Menezes, P. D., Gadegaard, N., Natal Jorge, R. M. and Pinto, S. I. S. (2021) Modelling human liver microphysiology on a chip through a finite element based design approach. International Journal for Numerical Methods in Biomedical Engineering, 37(5), e3445.

There may be differences between this version and the published version. You are advised to consult the publisher's version if you wish to cite from it.

This is the peer reviewed version of the following article:

Menezes, P. D., Gadegaard, N., Natal Jorge, R. M. and Pinto, S. I.

S. (2021) Modelling human liver microphysiology on a chip through a finite element based design approach. International Journal for Numerical Methods in Biomedical Engineering, 37(5), e3445, which has been published in final form at http://dx.doi.org/10.1002/cnm.3445

This article may be used for non-commercial purposes in accordance with Wiley Terms and Conditions for Self-Archiving.

http://eprints.gla.ac.uk/232836/

Deposited on: 28 January 2021

Enlighten - Research publications by members of the University of Glasgow http://eprints.gla.ac.uk 


\title{
Modelling Human Liver Microphysiology on a Chip Through a Finite Element Based Design Approach
}

\author{
P. D. Menezes ${ }^{1}$, N. Gadegaard ${ }^{2}$, R. M. Natal Jorge ${ }^{1,3}$, S.I.S. Pinto ${ }^{1,3^{*}}$ \\ ${ }^{1}$ Engineering Faculty, University of Porto, Rua Dr. Roberto Frias, s/n, 4200 - 465 Porto, Portugal \\ ${ }^{2}$ Division of Biomedical Engineering, James Watt School of Engineering, University of Glasgow, \\ University Avenue, Glasgow G12 8QQ, United Kingdom \\ ${ }^{3}$ Institute of Science and Innovation in Mechanical and Industrial Engineering, LAETA, Rua Dr. \\ Roberto Frias, 400, 4200 - 465 Porto, Portugal
}

\begin{abstract}
Organ-on-a-chip are microfluidic devices capable of growing living tissue and replicate the intricate microenvironments of human organs in vitro, being heralded as having the potential to revolutionize biological research and healthcare by enabling unprecedented control over fluid flow, relevant tissue to volume ratio, compatibility with high-resolution content screening and a reduced footprint. Finite element modelling is proven to be an efficient approach to simulate the microenvironments of organon-a-chip devices, and may be used to study the existing correlations between geometry and hydrodynamics, towards developing devices of greater accuracy. The present work aims to refine a steady-state gradient generator for development of a more relevant human liver model. For this purpose, the finite element method was used to simulate the device and predict which design settings, expressed by individual parameters, would better replicate in vitro the oxygen gradients found in vivo within the human liver acinus. To verify the model's predictive capabilities, two distinct examples were replicated from literature. Finite element analysis enabled obtaining an ideal solution, designated as liver gradient-on-a-chip, characterized by a novel way to control gradient generation, from which it was possible to determine concentration values ranging between $3 \%$ and $12 \%$, thus providing a precise correlation with in vivo oxygen zonation, comprised between 3-5\% and 10-12\% within respectively the perivenous and periportal zones of the human liver acinus. Shear stress was also determined to average the value of $0.037 \mathrm{~Pa}$, and therefore meet the interval determined from literature to enhance liver tissue culture, comprised between 0.01-0.05 Pa.
\end{abstract}

Keywords: Organ-on-a-chip, microfluidic devices, computational fluid dynamics, finite element analysis, oxygen concentration gradient, liver model.

*Corresponding author: e-mail: spinto@fe.up.pt; phone: (00351) 225081724 


\section{Introduction}

The pursuit to replicate human physiology in vitro has long been addressed as a primary ambition for study and research. It was approximately a century ago when conventional two-dimensional (2D) cell cultures were introduced, by the work of Harrison et al. [1]. Despite their established value, 2D cultures present significant limitations, as they fail to accurately replicate tissue-specific differentiated functions as known in vivo, as well as drug activities [2]. This was followed by the development of three-dimensional (3D) cell cultures, first reported by Ehrmann et al. [3], which provided a more accurate model to replicate the spatial and chemical complexity of living tissues. However, the difficulty of producing consistent structures, the lack of crucial environmental cues for cellular growth and the absence of fluid flow are amongst some of the major obstacles compromising the growth of the respective technology [2].

The opportunity to overcome these limitations has been recently introduced by organ-on-a-chip (OoaC) technology, a concept first attempted by Park et al. [4]. By merging microfluidics with tissue engineering, OoaC devices present continuously perfused chambers, of highly controllable and predictable conditions, in which cells are cultured to simulate tissue and organ-level physiology. Not only do these platforms provide microenvironments of greater relevance than conventional 2D and 3D culture systems, but also enable high-resolution, real-time screening of biochemical, genetic and metabolic activities of living cells within a functional organ context, established for an in vitro model [5]. Their miniaturized size further defines a reduced footprint, requiring only low amounts of reagents [6]. Due to its inherent qualities, OoaC technology is highly regarded to be of great potential to enhance the study of tissue development, organ physiology and disease etiology [5].

Pharmaceutical research is a field for which the application of OoaC technology is regarded to be of great benefit and relevance. The preclinical process for validation of potential new drugs is timeconsuming, extremely costly and inefficient [7]. It has been reported that, while investment towards the field keeps increasing [8], the number of drugs being approved by the Food and Drug Administration has constantly been declining [9]. This is believed to derive from the physiologically low relevance provided by preclinical methods, which commonly rely on animal models for the assessment of drug viability [7]. Ergo, OoaC devices emerge as highly appropriate candidates to replace conventional methods for an ethically accepted approach, that provides greater physiological relevance, higher throughput, and overall, improved efficiency for pharmaceutical research and development [10].

Drug-induced liver injury is a major concern for global human health, and it is one of the most common side effects of therapeutic compounds, an outcome which can be attributed to poor understanding towards the mechanisms underlying toxic response of the liver [11]. Leading a central role in human metabolism, the liver performs a multitude of functions to maintain whole body homeostasis [12]. Despite its physiological heterogeneity, conventional cell culture systems commonly 
lack to recapitulate crucial aspects of the liver microenvironment, such as the presence of metabolic zonation. This phenomenon ensures that various metabolic pathways operate in parallel in the most efficient manner, and it is known to be commonly lost during liver disease. Oxygen (02) gradients, as known within the liver acinus, are reported to be crucial modulators of metabolic zonation, and thus represent a key aspect towards replicating and understanding human liver microphysiology [12]. Zonation is characterized by three distinct regions, each defined by a specific interval of 02 concentration as follows: periportal zone, where blood is highly oxygenated, with an 02 tension of 60-70 $\mathrm{mm} \mathrm{Hg}$ [13] (approximately corresponding to $10-12 \%$ of 02 concentration [14]); transitional zone, characterized by intermediate 02 concentration values (5-10\% of 02 concentration [14]); perivenous zone, where blood is depleted of 02, with a tension of 25-35 $\mathrm{mm} \mathrm{Hg}$ [13] (approximately corresponding to $3-5 \%$ of 02 concentration [14]). However, fine-tuning 02 gradients to fully replicate this phenomena in vitro, remains currently as a challenging task to be addressed by OoaC applications [15].

The development of increasingly refined devices for purpose-specific application becomes then an appealing ambition, which has engaged the research community to extensively explore the field of OoaC technology [16]. Finite element analysis is well-established as a powerful tool for the simulation and study of a wide range of biomedical applications [17,18], such OoaC, by providing detailed information regarding the governing physics and respective properties characterizing the multidisciplinary behaviour of these miniaturized devices $[6,14,19,20]$. Ergo, correctly employing finite element analysis has the potential of leading to a more efficient development of increasingly specific OoaC devices, without requiring the common experimental procedure of trial and error, known to be both time-consuming and generate high quantities of material waste. Geometric parameters are widely expressed by analytical microfluidics to perform crucial roles on determining the fluidic regime [21]. Therefore, even if insufficiently addressed by literature, such correlation is prone to represent an efficient strategy towards developing $\mathrm{OoaC}$ devices of greater specificity and functionality, one that can be easily explored and studied through a finite element approach.

The present study intends to go further on analysing the existing correlations between design parameters, such as channel dimensions, flow rate, and resulting microenvironment, for the refinement of a microfluidic device, modelled through a finite element approach based on COMSOL Multiphysics ${ }^{\circledR}$ modelling software. The device aims to better simulate, in vitro, 02 zonation as found in vivo within the human liver acinus, by providing controlled long-term gradient generation over a cell culturing site. Its design principles are inspired by Jeon et al. [22], where an array of serpentine-like channels function as a premixer [23], meant to enable gradient generation by establishing repeated splitting, mixing and recombination of various fluid streams. Through the employment of a finite element approach, the present work aims to re-adapt the respective device for OoaC applications, by studying and refining its respective design parameters. An initial design, providing the purpose of generating concentration gradients, analogous to the reported by Jeon et al. [22] (Figure 1 (a)) but 
within a simplified arrangement of just two inlet channel, for the input of respectively two different concentrations only, is designated as gradient-on-a-chip (GoaC) and illustrated in Error! Reference source not found. (b). This device will be modelled and refined in the following, towards producing gradients of 02 that more accurately replicate the microenvironment

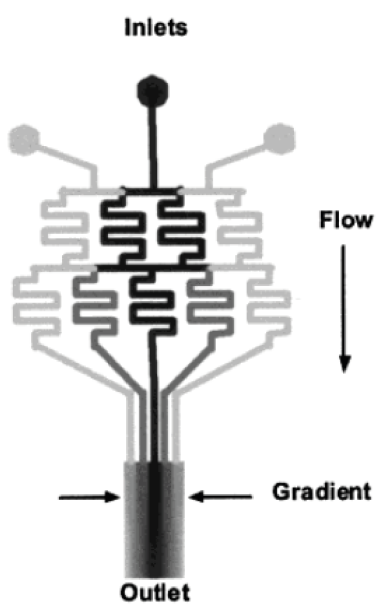

(a)

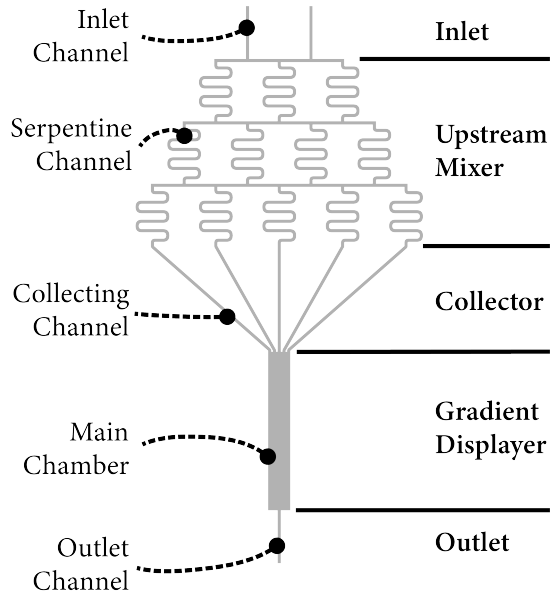

(b)

Figure 1: (a) Design introduced and tested by Jeon et al. [22]. Figure is reproduced with permission from Jeon et al. [22]; (b) The GoaC as initially modelled, with domains identified on the left, and sections on the right.

found in vivo within the human liver acinus. The obtained solution is expected to establish a more relevant liver model for $\mathrm{OoaC}$ for OoaC applications, by providing an environment of greater physiological resemblance for the growth of liver organoids - cellular constructs envisioned to contribute with properties of high biological pertinence [24]. The finite element approach is intended to enhance the design refinement process of the $\mathrm{OoaC}$ device, by establishing a platform for studying the intrinsic multi-disciplinary relations characterizing the respective microfluidic solution. The model's predictive capabilities are to be validated by replicating examples provided by literature, thus legitimizing the relevance of the developed platform towards studying, simulating and refining OoaC applications.

\section{Methodology}

Finite element modelling of the $\mathrm{GoaC}$ was performed using COMSOL Multiphysics ${ }^{\circledR}$ modelling software. Numerical models have been set to simulate the multiphysics nature of OoaC applications, by engaging modules describing the necessary fluidic and chemical dynamics. The developed framework was further entailed to be both parametric and modular, thus enhancing its potential to study and manipulate the effect of geometry on the established fluidic microenvironment. Mesh was designed and refined to specifically meet the characteristics of the $\mathrm{GoaC}$ device, by targeting the critical domains for simulation whilst reducing the associated computational time. To legitimize the model's predictive capabilities and accuracy, two distinct applications provided by literature were replicated, covering separate fields of relevance for the present application. 


\subsection{Fluidic Analysis}

Microfluidic systems introduce a paradigm change when compared to common fluidic applications, requiring therefore a likewise exclusive method for analysis. Reassessing the continuum hypothesis indicates that, for microchannels, the sensitive volume sample in analysis is large by comparison with a fluid particle, determining a continuum state for the properties of the fluid and ensuring that the ordinary hydrodynamic equations may be employed [21]. However, by performing a dimensionless analysis, it is acknowledged that common volume forces, such as gravity and inertia, become largely irrelevant within these miniaturized devices, whereas surface related forces, such as surface tension and viscosity, acquire a widely dominant influence on determining the underlying dynamics [25].

The Reynolds number, $R e$, defined by the ratio between inertial and viscous forces, is commonly employed to determine the fluidic regime associated with a given application. It is described as follows:

\section{$R e=\rho U D h \mu$}

where $\rho$ is the fluid density, $U$ is the flow velocity, $D h$ is the hydraulic diameter and $\mu$ is the fluid dynamic viscosity. Microfluidic systems, such as OoaC applications, being commonly described by low fluid velocities and small channel dimensions, are accordingly characterized by a low Reynolds number, usually neighbouring the value of $R e \approx 1$ [21]. This reveals the laminar nature of the fluidic regime within such devices [22,26,27], a condition characterized by deterministic streamlines where fluid velocity presents a single component, oriented along the channel's length [28].

The laminar character further dictates that, between two streams of laminar flow in contact with each other, mixing of particles occurs only by diffusion [29]. Therefore, for OoaC applications, two distinct timescales become relevant to analyse mixing of a given diluted species between streams of laminar flow distributed along a particular domain [25]. These are the time for convection, $t c w$, and the time for diffusion, tdiff, expressed as follows:

tconv $=l U$

tdiff $=w 2 N f 2 D$

where $l$ is the length of the channel, $w$ is the width of the channel and $N f$ is the number of flow streams and $D$ is the diffusion coefficient rate. By having tconv «tdiff, it means that diffusion will not have enough time to occur, and therefore, the solution will largely remain unchanged. However, 
for the opposite case, where tconv $\geq \operatorname{tdiff}$, diffusion will achieve a steady state before the end of the domain in analysis, characterizing a state of full mixing.

\subsection{Modelling of Fluid and Particle Dynamics}

The present finite element approach was defined by engaging two fundamental interfaces, designated as Computational Fluid Dynamics (CFD) and Transport of Diluted Species Modules, solving respectively for the intrinsic fluid dynamics and particle motion of both solvent and solute, and applied to a particular set of boundary conditions and testing features, to define both the device and the specific environment required for operation. The established method is described in the following.

\subsubsection{CFD Module}

The dynamics governing fluid motion were engaged by employing COMSOL's CFD Module, to solve the system in regard to the Navier-Stokes equation, accounting for conservation of momentum, coupled with the continuity equation, to impose conservation of mass accordingly [30]. Furthermore, the solver was set to analyse the steady-state solution, and therefore simulate the device's purpose of generating long-term gradients $[22,23,26]$. Subsequently, the Navier-Stokes equation is expressed as follows:

$\rho \mathbf{u} \cdot \nabla \mathbf{u}=-\nabla \cdot p \mathbf{I}+\nabla \cdot \mu \nabla \mathbf{u}+\nabla \mathbf{u} T-23 \mu \nabla \mathbf{u I}+\rho \mathbf{F}$

where $\mathbf{u}$ is the velocity vector, $p$ is the fluid pressure, $\mathbf{I}$ is the identity matrix and $\mathbf{F}$ are the volume forces per unit mass of fluid. The continuity equation, on the other hand, is defined as:

$\nabla \cdot(\rho \mathbf{u})=0$

\subsubsection{Transport of Diluted Species Module}

To predict particle motion within the bulk flowing medium, the transport mechanisms of advection and diffusion must be engaged and coupled. Such is enabled by employing COMSOL's Transport of Diluted Species Module. This interface engages the advection-diffusion equation, deriving from Brownian motion and Fick's law [21,31], to solve for the combined effect of both transport mechanisms on a given species diluted within a specific medium. According to fluid motion, the solver will be likewise set to analyse the steady-state solution, from which the advection-diffusion equation may be written as follows:

$\mathbf{u} \nabla C-D \Delta C=s(x)$ 
where $C$ is the species concentration, $s$ is a source/sink of the respective species and $x$ is the position along the channel. This equation will be applied to a particular set of boundary conditions, defined in the following, and solved to calculate the concentration levels of the given diluted species, within the domains of the device and according to the velocity field defined at the CFD Module.

\subsection{Boundary Conditions and Parameters}

To compute a solution for the given system of equations, specified previously to describe fluid motion and mass transport, the method requires the establishment of an additional set of constraints, known as boundary conditions, to fully define the present numerical framework.

For fluid dynamics, and within the CFD Module, both inlet channels were defined to provide a constant and equal flow rate $(\mathrm{Q}$ in $)$ of media. The outlet channel was expressed as being the exit port for media flow [22]. The remaining boundary conditions were simulated as impermeable walls, with a no-slip boundary condition [32]. Finally, media was modelled to be water by defining its density and dynamic viscosity according with the values presented in Table 1 .

Following a similar process, the Transport of Diluted Species Module was engaged, by defining oxygen as the dissolved species and simulating its physiological behaviour within the flowing media. Inlet channels were described separately, with the left inlet defined as the only supplier of the given diluted species. The concentration supplied at the inlet was defined as being the 02 concentration in water at the testing temperature $37 \stackrel{\circ}{\circ}$ [14], which accounts for media containing $18 \%$ of dissolved 02 $[33,34]$. The outlet channel was modelled as being the exit port [22], while the remaining boundaries were defined as impermeable walls, preventing further exchange of 02 between the system and its surroundings. The diluted species was simulated as being 02 , with a diffusion coefficient rate, within the given media and at the respective testing temperature, as presented in Table 1.

\section{TABLE 1}

\subsection{Parametric and Modular Based Modelling}

The present project intends to determine the ideal conditions for generating liver 02 zonation within the $\mathrm{GoaC}$ device, for which geometric components, as expressed by analytical microfluidics, play a crucial role. Therefore, to provide a deeper understanding of the correlation existing between geometry and established fluidic microenvironment, the method was developed to be both parametric and modular, respectively meaning that every dimension was defined as being an independent parameter, open for user manipulation, and all the geometric entity were modelled individually, based on their own set of parameters, stored separately within a parts library available through the software, and open to be assembled within the main model. To maintain geometric coherence for every given combination of parameters and modular entities, it was required the development of a specific language to distinguish between structures and enable their collective assembly within the respective 
workspace, achieved by connecting parameters within developed expressions, particular to each individual structure, as exemplified by the expression below:

Where SAStPt $X$ represents the distance along the $x$ axis where the upstream mixer is placed, $S w c$ stands as the width of the serpentine channels, $S A d b s$ is the distance between serpentines, $S A d b o c$ represents the distance between the outlets of the collecting channels and SAwoc their respective width. From both approaches, it was intended to create a dynamic method to improve the design refinement procedure of the GoaC, towards promoting a faster and deeper understanding of how geometric parameters affect gradient generation, either through their individual or collective influence. By changing parameters, this method allowed to obtain various geometries within the same model. For mere illustrative purposes, three distinct geometries are presented in Figure 2 (b), (c) and (d) by randomly changing parameters as those shown in Equation (7), towards demonstrating the model's geometric flexibility. Following a similar methodology, datasets were implemented specific to each geometric module and based
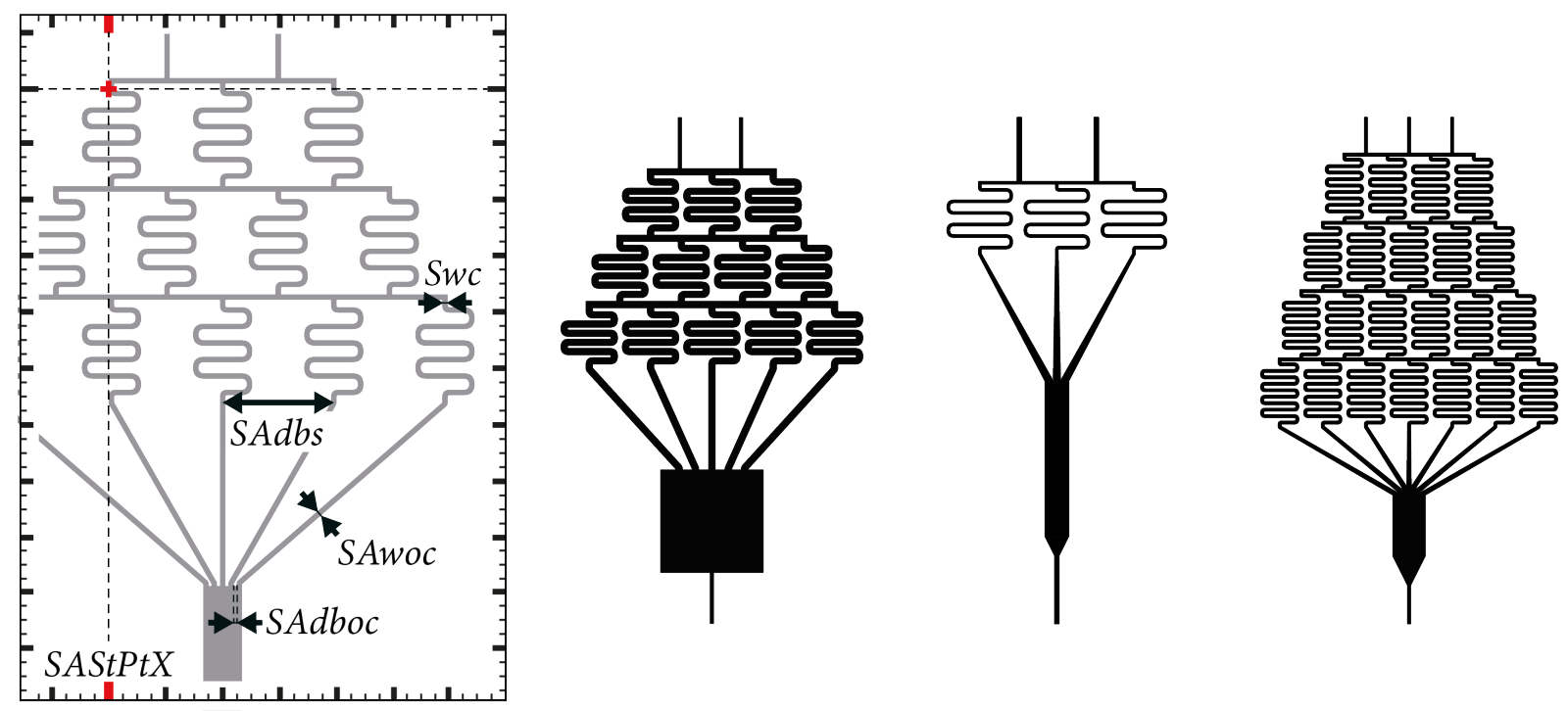

Figure 2: Illustrative examples, generated by randomly modifying parameters, to demonstrate the model's geometric flexibility conveyed by the developed parametric approach. (a) Initial design, augmented to demonstrate the parameters introduced by equation (7), which respectively assume the following values:

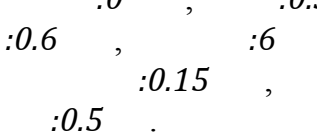

(a)

(b)

(c)

(d) 
parameter-based relations, automatically adapting to the domain they are implemented in. The main goal being the establishment of gradients of 02 at the main chamber, a $7 \times 5$ probe grid was established within the respective domain to provide a constant and quantifiable way to measure and assess the quality of the gradients being generated, as seen in Figure 3 (a). Vertical lines are designated as Concentration Lines (CL), while horizontals are denominated as Gradient Lines (GL). Furthermore, and given the crucial role played by the serpentine channels at the upstream mixer, datasets were likewise implemented within the respective domain, by establishing probes transversal to flow direction, called Serpentine Lines (SL), visible in Figure 3 (b), and therefore assess the concentration variation along these channels.

(a)

\subsection{Mesh Convergence Analysis}

Establishing a mesh convergence analysis is crucial for the development of any given finite element
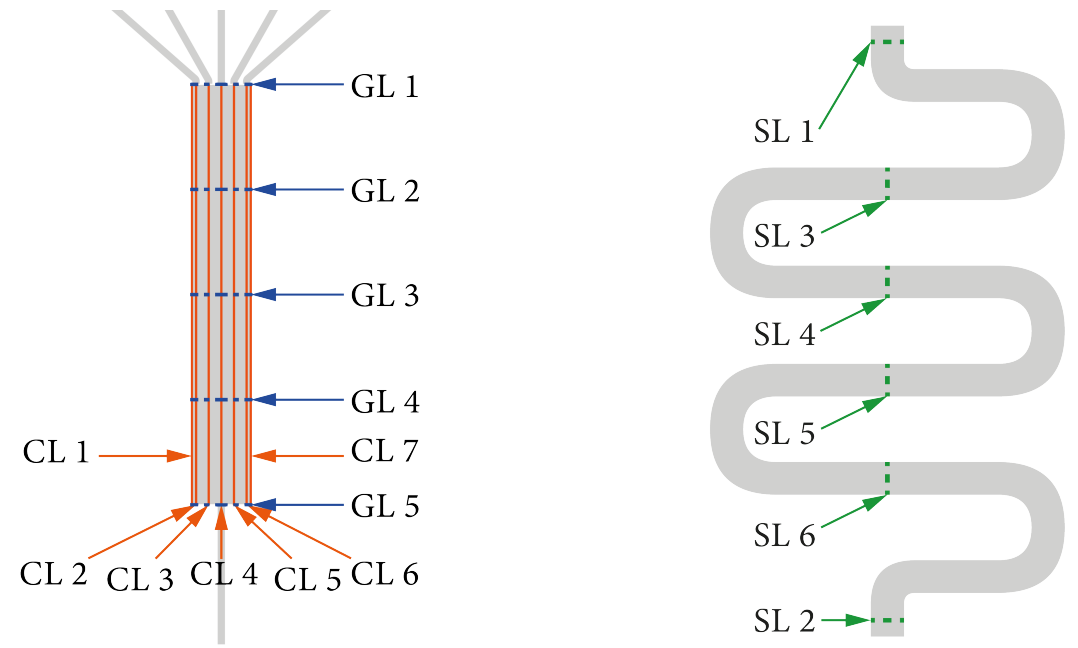

application. It provides knowledge regarding the necessary element resolution to guarantee mesh independent results, and therefore, ensure a balance between accuracy of result outcome and efficiency of computational time in determining a solution. To perform a mesh convergence analysis on the present model, $\mathrm{O} 2$ concentration levels were taken into consideration, as they must not exceed the concentration supply at the inlet (18\%). Therefore, this value was used as a reference to assess the quality of the mesh in regard to the accuracy and legitimacy of the results being generated. Each domain, within the device, will be set to return their respective value for the respective quantity, and subjected to an individual analysis.

Initially, a study was performed by employing a homogeneous mesh within the model, followed by a constant increase of the total number of meshing elements, as seen in Figure 4 (a). The purpose was to obtain the minimal number of elements, to be uniformly applied throughout the model, required to ensure the computation of mesh independent results, which, for the present study, was translated by maximum oxygen concentration values approaching and remaining below the value of $18 \%$. From a coarser resolution, defined by a total number of 28572 elements (Point A, Figure 4 (a)), mesh was gradually improved until the achievement of an extremely refined mesh, characterized by a total of 
858011 meshing elements (Point B, Figure 4 (a)), for which the software calculated mesh independent results, with 02 concentration levels remaining below the reference value of $18 \%$. Computational time ranged from $19 \mathrm{~s}$, for the coarser mesh, to a total of $5594 \mathrm{~s}(1 \mathrm{~h} 33 \mathrm{~m} 14 \mathrm{~s})$, for the finer mesh. By performing the previous study, not only was it possible to set the standard for mesh quality, but also identify the domains which are most critical for simulation, and therefore, requiring a finer mesh. These domains were acknowledged to be the main chamber and the initial sections of the inner serpentine channels, with respective minimal number of elements of 239837 (Point C1, Figure 4 (b)) and 192432 (Point C2, Figure 4 (c)), achieved for the finer mesh. As for the remaining domains, the study suggested mesh could be relaxed individually whilst maintaining result accuracy, to therefore achieve a customizable mesh of increased efficiency. The obtained solution, designated as the ideal mesh, exhibited in Figure 5, is characterized by a decreased total number of elements, equivalent to 553091 (Point O, Figure 4 (a)), whilst maintaining the minimal element resolution established previously for both critical domains. The present mesh constitutes a model of greater time-efficiency, requiring only a total of $295 s$ to compute a solution of high accuracy and legitimacy. Elements within the mesh were defined to be predominantly triangular, but contemplating further refinement at the boundaries, ensured by shallow quadrangular shaped elements (Figure 5 (d)), to resolve thin boundary



layers for fluidic applications, such as the present, at the no-slip boundaries.

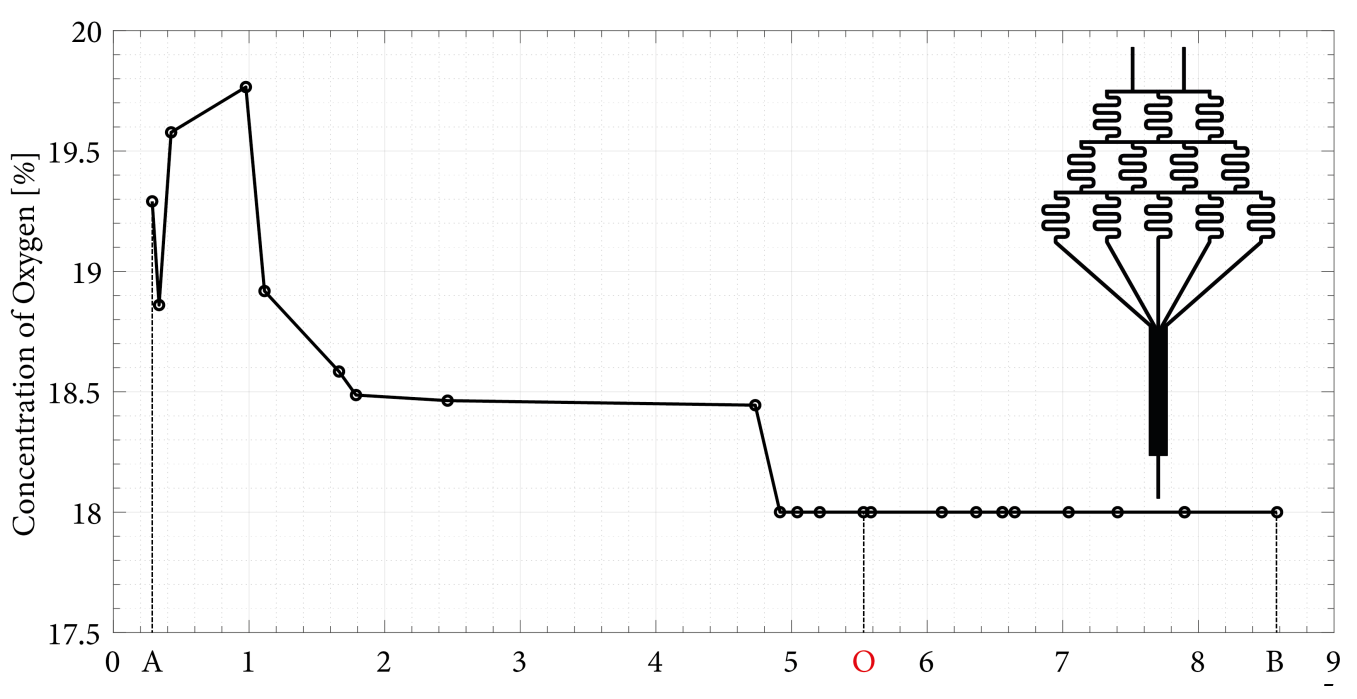






(b)

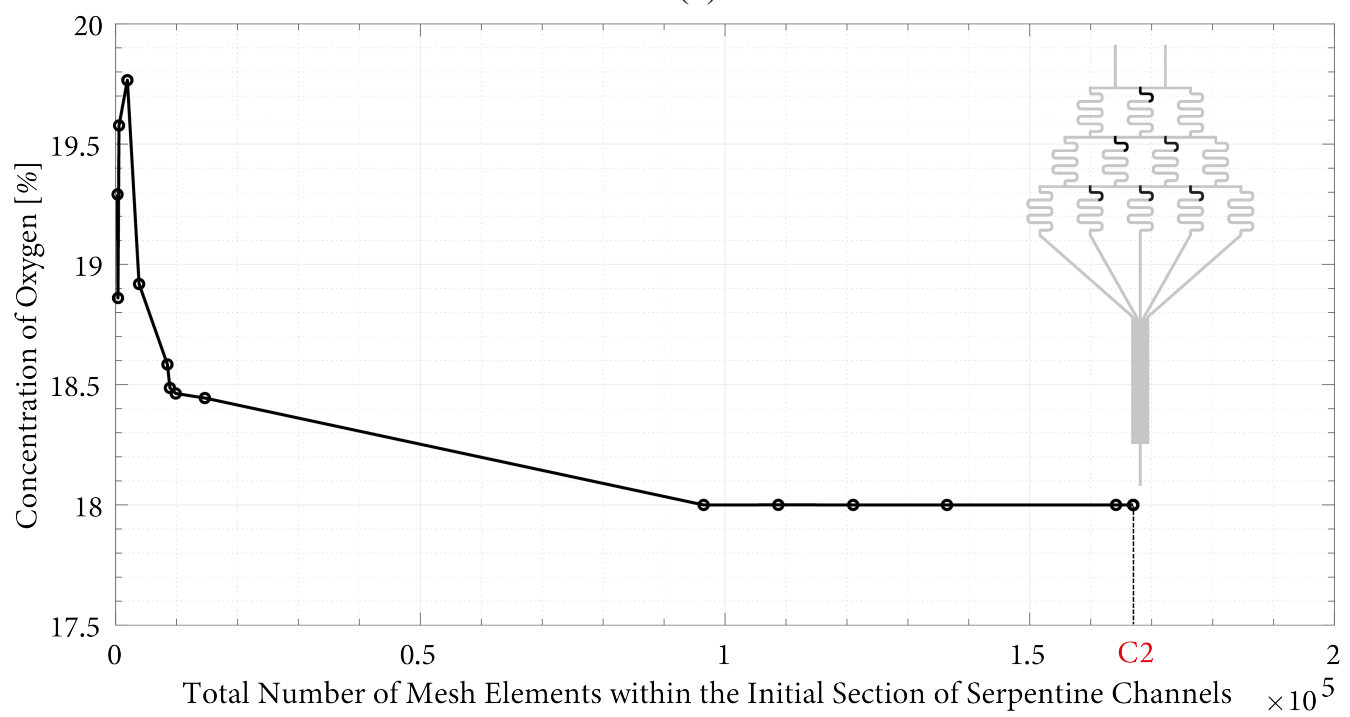

(c)



(a)



(b)

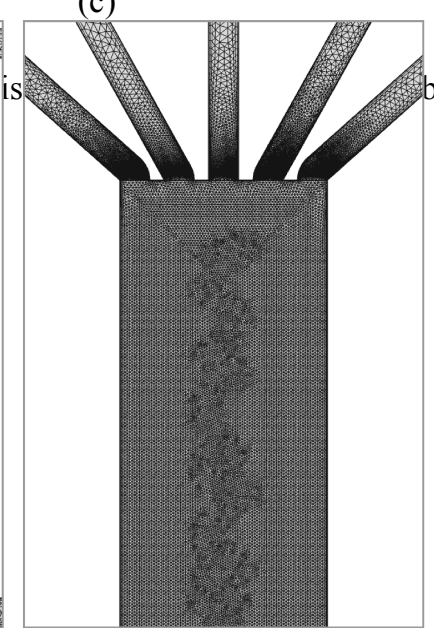

(c)



(d)

Figure 5: (a) Ideal mesh, highlighting the refinement towards (b) the initial section of serpentine channels and (c) the main chamber, as well as exhibiting (d) the boundary layer mesh. 


\subsection{Verification of the Model's Predictive Capabilities}

Having the framework for finite element analysis fully set, it is common practice, and a crucial procedure, to assess the legitimacy of its predictive capabilities before putting the model into use, by replicating experimental results as reported within literature. In the present work, such was achieved by simulating two different examples, each aiming to address a distinct features essential for performing the study the present work is compromising to achieve. The respective examples, presented in the following, are then intended to provide a means through which it is possible to understand how precisely the model replicates real-world data.

\subsubsection{Example 1: Verifying Gradient Generation}

Generating gradients is the key function of the GoaC device, and therefore, legitimizing the model's capability to simulate such phenomena is of fundamental importance. The work of Jeon et al. [22] has pioneered on introducing the steady-state gradient generator, whose design principles are followed by the device addressed in the present paper. Hence, replicating the experimental results reported by Jeon et al. [22] was regarded as a procedure of utmost importance to verify the legitimacy of the established finite element approach on predicting gradient generation.

By taking advantage of the modular and parametric capabilities of the model, the literature referenced design was replicated. Furthermore, parameters have been set to simulate the experimental conditions reported by the respective reference for Gradients in Solution, for which solute and solvent were modelled as being respectively fluorescein isothiocyanate in a sodium bicarbonate buffer, with parameters as expressed in Table 2. Inlet Flow Velocity (Uin) was tested for three different magnitudes, and equivalent to $1 \mathrm{~mm} / \mathrm{s}, 10 \mathrm{~mm} / \mathrm{s}$ and $100 \mathrm{~m} \mathrm{~m} / \mathrm{s}$.

Simulations were carried to obtain both fluorescence micrographs of solution gradient and the gradient profile at $500 \mu \mathrm{m}$ downstream in the main chamber, and therefore, enable comparison between literature experimental results and numerical data obtained from the developed method. Both simulations achieved great affinity, as seen in Figure 6 between the literature referenced fluorescence micrograph ((a), (b) and (c)) and the numerically obtained concentration distributions ((d), (e) and (f)), as well as for the gradient profile $((\mathrm{g}),(\mathrm{h})$ and (i)). Due to the precision exhibited by the computed results on replicating literature results, the present example stood as a main argument to legitimize the model's capability on predicting gradient generation.

\section{TABLE 2}




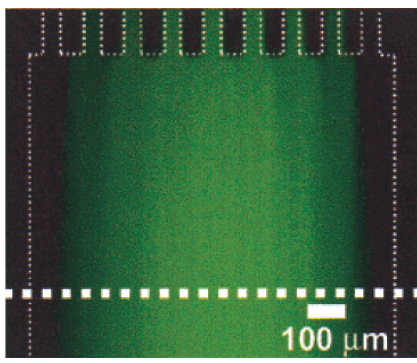

(a)

(d)

(g)

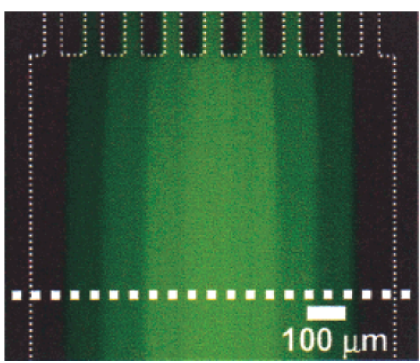

(b)

(e)

(h)

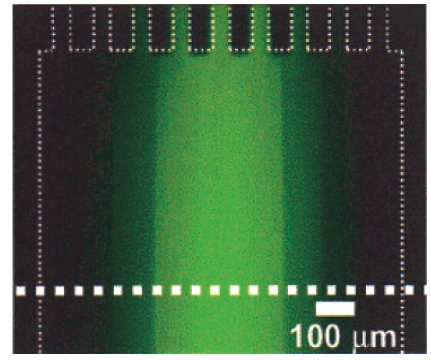

(c)

(f)

(i)

Figure 6: Comparison between (a), (b) and (c) fluorescence micrographs reported by Jeon et al. [22] and (d), (e) and (f) obtained numerical concentration distribution profiles, as well as (g), (h) and (i) overlapped gradient concentration profiles for analytical data (black dots), literature reported experimental results (black line), both reported by Jeon et al. [22], and numerically computed data (yellow line) from the present model being developed. Figures are reproduced with permission from Jeon et al. [22].

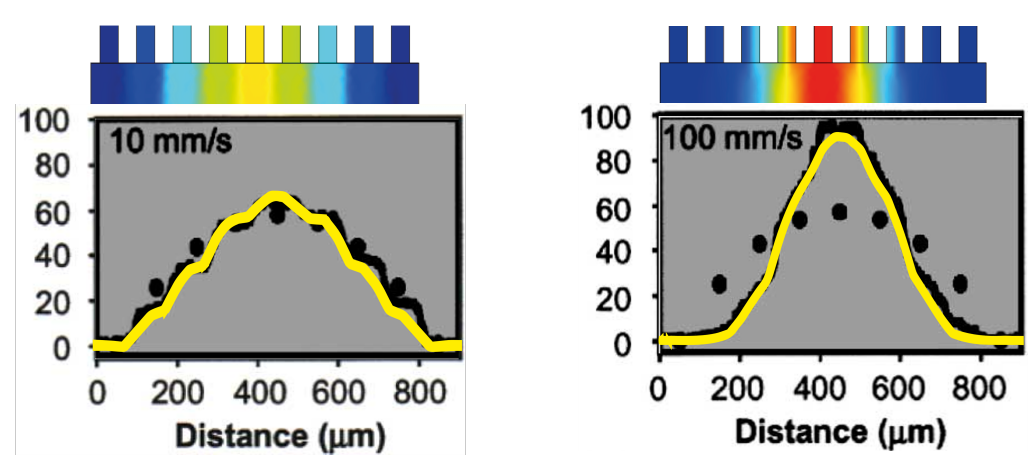

\subsubsection{Example 2: Verifying Shear Stress}

Shear stress is described by literature to have a significant effect on cellular culture and function [3539]. Ensuring cells are being subjected to controlled and specific values of shear stress is crucial to stimulate their respective activity, which therefore demands OoaC applications to carefully approach the present topic. The work of Tanaka et al. [35] evaluates the effect of shear stress on a culture of liver metabolic cells designated as hepatocytes, by changing either the dynamic viscosity of the media being perfused or the Qin. Due to the focus on liver specific cells and respective response to the determined shear stress microenvironment, the results describing the correlation between dynamic viscosity, Qin and shear stress were modelled by using the present finite element approach.

The microchannel used for experimental testing was characterized by a rectangular section, with dimensions of $0.25 \times 0.1 \mathrm{~mm} 2$ for respectively the width and height, and a total length of $40 \mathrm{~mm}$. Four distinct experiments were reported. Initially, the medium's dynamic viscosity was maintained at the 
constant value of $12 \mathrm{mPa} \cdot \mathrm{s}$ while $Q$ in changed between the values of $1 \mu \mathrm{L} / \mathrm{min}$ and $10 \mu \mathrm{L} / \mathrm{min}$. In a different setting, $Q$ in was maintained at the constant rate of $2.5 \mu \mathrm{L} / \mathrm{min}$, while the medium's dynamic viscosity shifted between $1 \mathrm{mPa} \cdot \mathrm{s}$ and $12 \mathrm{mPa} \cdot \mathrm{s}$.

Initially, a 2D model of the reported channel was modelled and used for obtaining results. However, shear stress values were presented with a significant discrepancy between literature and numerical results (see Table 3). It was therefore acknowledged that, for shear stress prediction, the effect of upper and bottom walls should not neglected, especially for shallow channels as the one reported, which therefore suggested the implementation of a $3 \mathrm{D}$ model. This solution was capable of predicting shear stress with greater accuracy, even if still presenting slight fluctuations in comparison with literature data. Results for shear stress may be overviewed in Table 3.

TABLE 3

\section{Results and Discussion}

Having the framework for finite element analysis fully developed and verified, it followed its application for refinement of the $\mathrm{GoaC}$ device, towards developing a more relevant liver model by modifying its features to produce gradients of 02 accurately simulating the values found in vivo within the human liver acinus.

First, a brief analysis will be established to analyse the relative influence of each individual parameter on gradient generation. Such an assessment intends to create a clearer understanding of how various parameters regulate the behaviour of the $\mathrm{GoaC}$, as well as establish a design procedure to guide the following refinement process of the respective device. Features will be defined to enhance 02 gradient generation at the main chamber, by creating a uniform and well-defined environment of predictable $\mathrm{O} 2$ concentration levels meant to match the values of liver zonation as found in vivo and ensure correct liver tissue culture and function at the respective domain. The final and enhanced solution, obtained after feature refinement, is designated as liver gradient-on-a-chip (LGoaC) device.

\subsection{Overview of Parameters}

The present finite element method is described by a broad set of parameters, defining physics, material selection, study options and design features. Parameters expressing both solvent and solute will be considered as constants throughout the design refinement procedure, accounting for the perfusion of an unchanging solution of media and diluted species. The variables considered for testing and analysis will be related to design features, being pondered and defined together with the employed Qin, to fully evaluate each parameters and define their respective influence on fluid dynamics and subsequent gradient generation.

An initial assessment was carried towards providing a clearer understanding in regard to the individual effect on gradient generation performed by each parameter. This study enabled the establishment of a 
hierarchical picture regarding every parameter, and sustain a design sequence to carry the refinement process of the GoaC device, to be carried in the following. Qin was acknowledged to be the main variable for gradient development, while design features were interpreted as purely means for refinement. The effect of each parameter and their respective grade of influence on gradient development may be seen in Table 4. Due the high dependency on the applied Qin, the design refinement procedure was determined to consist in: first, establishing a fitting Qin interval; second: studying and refining all the design variables, as a function of the applied Qin interval, towards generating gradients of 02 with greater physiological relevance; third: defining a single $Q$ in value to ensure most relevant $\mathrm{O} 2$ gradient generation. Because the fluidic and chemical state of any given point within the device was studied to be compromised by the respective environments of anterior points, the design refinement process was further characterized to consist of a top-down approach, where geometric parameters are studied and defined following the direction of flow, from inlet to outlet channels.

\section{TABLE 4}

\subsection{Initial Iteration for Inlet Flow Rate}

Qin was acknowledged as the major parameter to control diffusive mixing and therefore, gradient development at the main chamber. Due to the high dependency of the device's behaviour in regard to the respective variable, an interval of most relevance to the current application will be defined, from which geometric parameters will be determined in respect to.

Simulations were performed to test a wide interval of Qin, ranging from Qin to Qin, with steps of order 10 in between. From the results presented in Figure 7, it was determined that, to obtain a fullgrown gradient uniformly developed along the length of the main chamber, the ideal Qin interval should be found for magnitudes above Qin. Such value was considered as the lower limit of the interval, whereas the upper boundary was defined in regard to literature, towards determining a value already accepted and employed for other applications of within microfluidics. Within literature, devices meant for gradient generation employ a diversified range of Qin. The work of Jeon et al. [22] tested three distinct values of Uin, corresponding to an Qin of $18 \mu L / h, 180 \mu L / h$ and $1800 \mu L / h$, whereas the Multi-Purpose Microfluidic Perfusion System developed by Gregory at al. [40] employed an $Q$ in equal to $240 \mu L / h$ and $2640 \mu L / h$. Given the high disparity, it has been selected the highest employed Qin for gradient generation within microfluidic systems reported by literature, and approximated to $2.5 \times 103 \mu L / h$, representing the upper limit of the Qin interval found as most 
suitable for the design process of the GoaC. This interval was then ultimately determined to be comprised within $1 \times 103 \mu L / h$ and $2.5 \times 103 \mu L / h$.

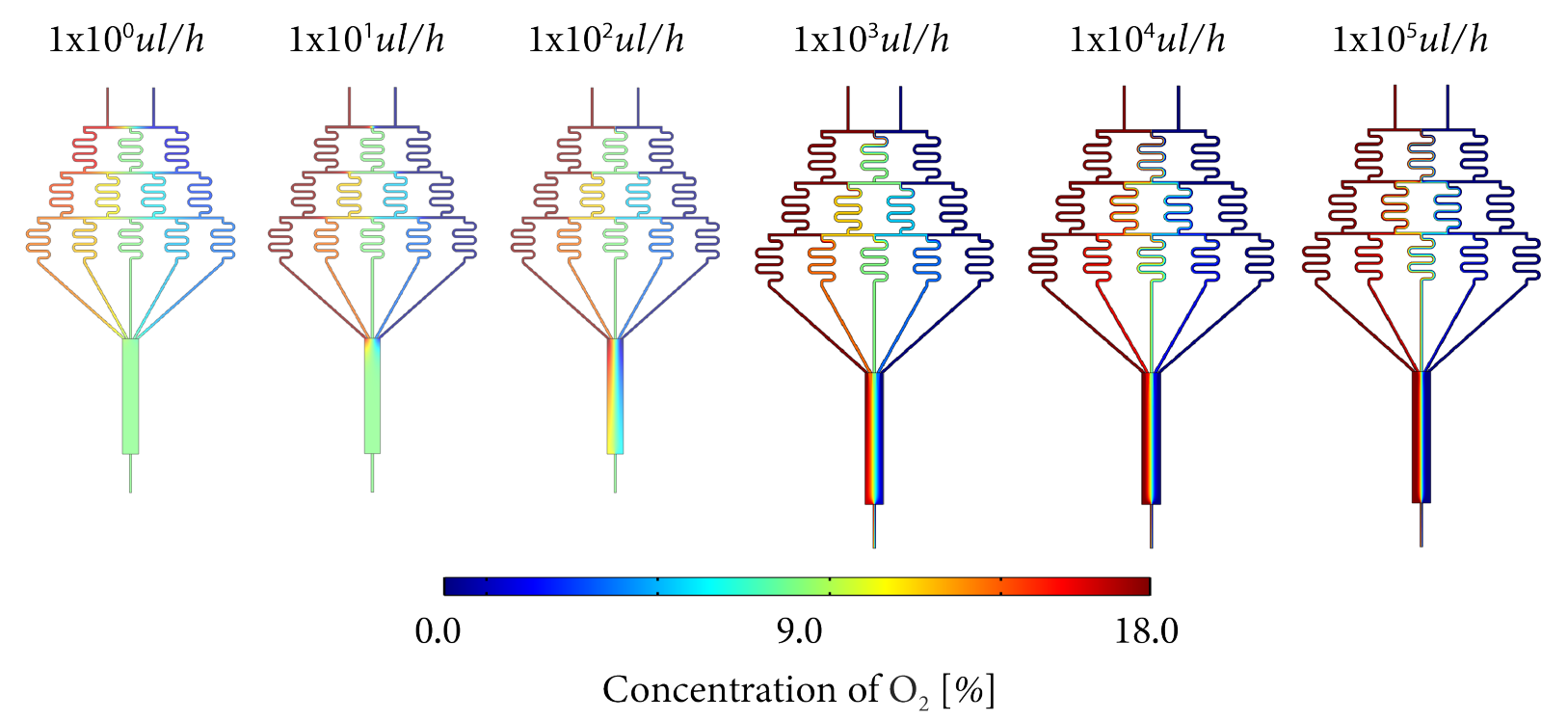

Figure 7: Influence of Qin in generating 02 concentration gradients at the main chamber of the GoaC device.

\subsection{Serpentine Channels}

It was defined a Qin interval of high order to enhance gradient development at the main chamber by reducing tconv. However, the same effect will be imposed at the serpentine channels, where the purpose is rather to fully mix the species carried by the two streams of flow that come into contact, meaning that due to the high Qin, this function was compromised for the initial design, as seen in Figure 8 (a). Such suggested a required readjustment for the serpentine channels. The design target behind modifying the respective domains is to therefore ensure full mixing after each channels, which can be translated by the condition tconv>tdiff, and achieved by either augmenting tconv or tdiff. As denoted in Table 4, the width of the serpentine channels must be considered with care in regard to organoid input, to avoid constraining the passage of these constructs towards the culturing site, here the main chamber. The liver organoids planned to be used within the present application possess a diameter of $0.25 \mathrm{~mm}$, which therefore suggested the width of the channels should be kept at $0.3 \mathrm{~mm}$, as contemplated by the initial design of the GoaC, to therefore allow easy flow of organoids, without constraints. With the width, and therefore tdiff already restricted, the goal was to increase the tconv towards enabling full mixing of species after each channel. Therefore, the total length of the serpentine channels was extended to $31.78 \mathrm{~mm}$ and the number of serpentine loops was increased to 4 , from 
which it was possible to maintain 02 concentration levels after each channel below a variance of $1 \%$, as it is possible to observe through Figure 8 (b).
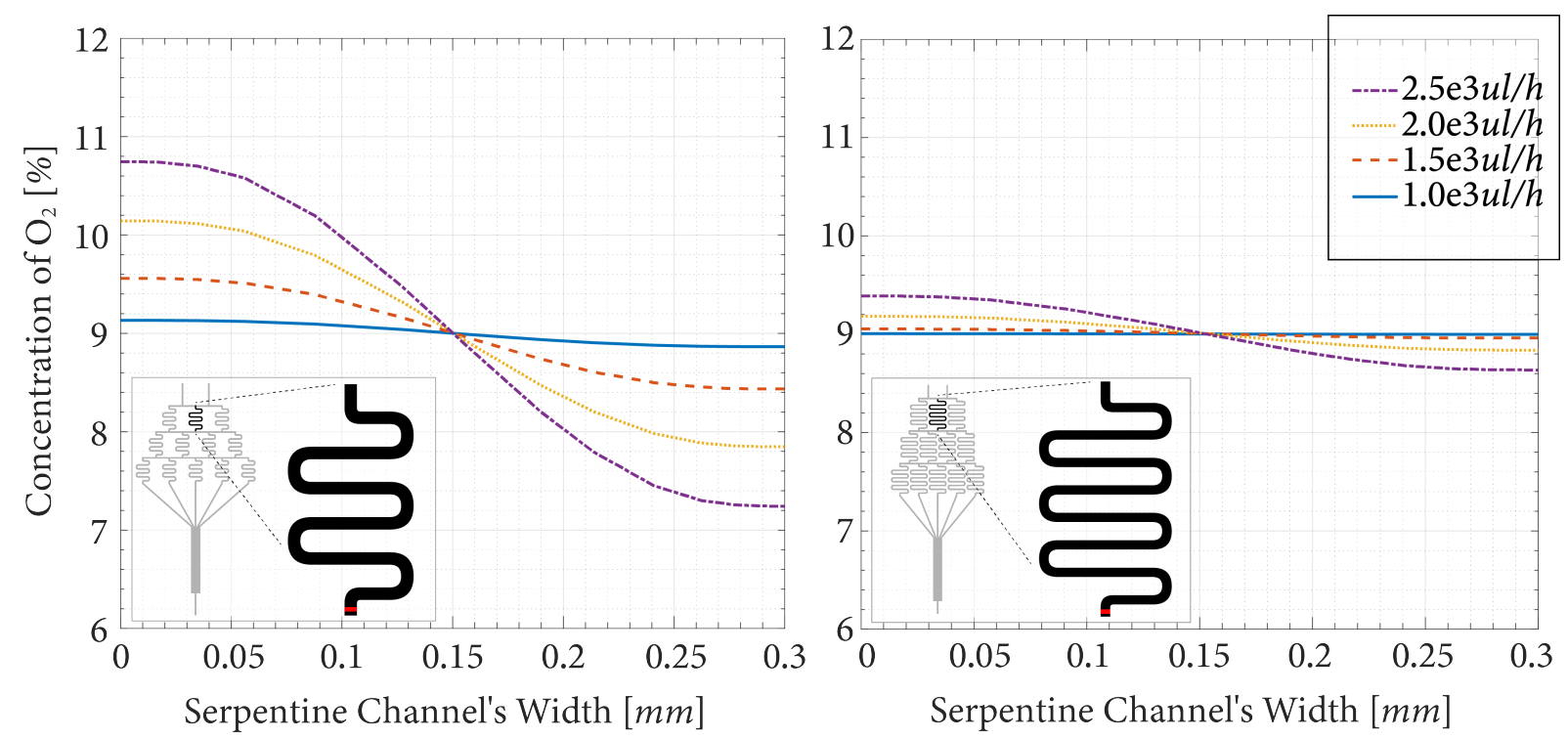

Figure 8: Concentration gradient at SL2 for both (a) initial and (b) improved serpentines at the GoaC device.

(a)

(b)

\subsection{Collecting channels, main chamber and outlet}

The domains comprised by both collecting channels and main chamber are defined by five major variables, four of which were acknowledged to be of relevance for gradient refinement, as seen in Table 4. The goal behind refining the respective geometric entities is to enhance gradient development at the main chamber, by attempting to create a steadier and highly uniform microenvironment, and subject liver organoids to more predictable and controllable 02 concentration levels. Such target can be translated by the requirement of tconv<tdiff.

The first ambition was to increase tdiff by augmenting the width of both domains, which as expressed by Equation 3, has a direct correlation to the time the diluted species possesses to diffuse across a given channel. Simulations further indicated that the parameters regulating tdiff, these being the width of both collecting channels and main chamber, as well as the distance between collecting channel's outlets, should be considered and refined accordingly, to ensure the correct achievement of steadier CLs resulting from increasing tdiff, and avoid the creation of highly disordered flow profiles, as seen in Figure 9 (a) and (b). Therefore, both width of collecting channels and main chamber were increased, respectively to be equal to $0.5 \mathrm{~mm}$ and $3.1 \mathrm{~mm}$, while the distance between collecting channel's outlets was kept at $0.15 \mathrm{~mm}$. The respective arrangement (illustrated by Figure 9 (c)) enabled reducing the $\mathrm{O} 2$ concentration fluctuations existing along each $\mathrm{CL}$, as demonstrated in Figure 
10 (a) for the initial design, to generate a much steadier environment. By shortening the main chamber
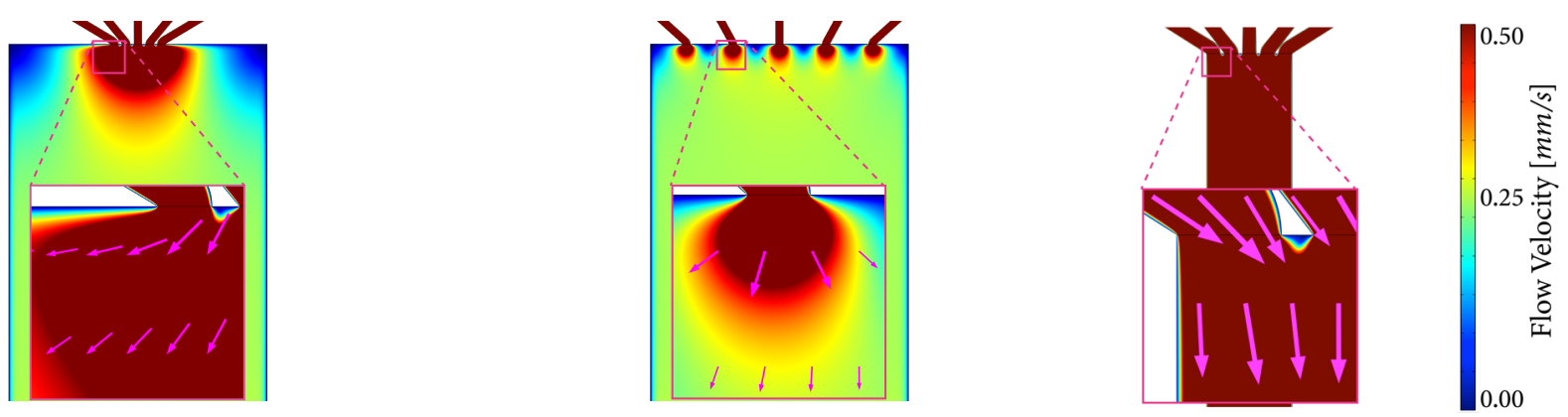

Figure 9: Flow profile and array field for multiple configurations for main chamber and collecting channels.

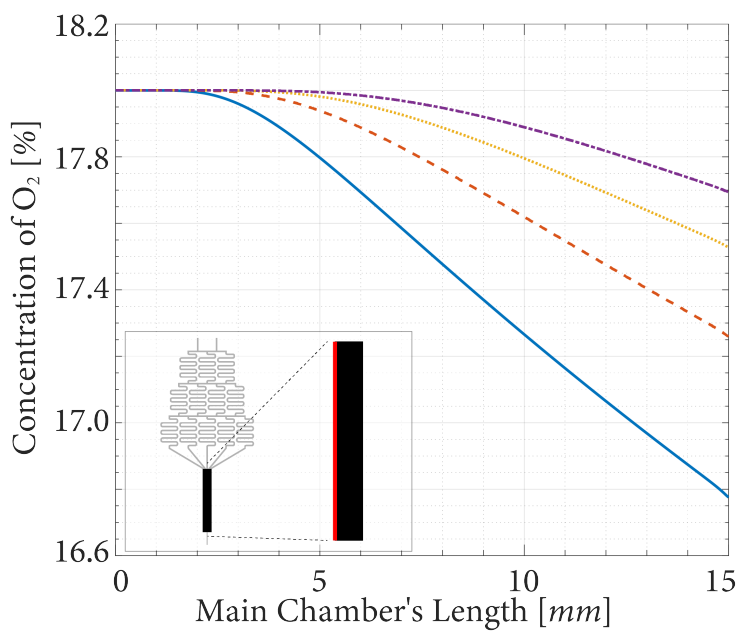

(a)



(b) (c)

(a)

(b)

tconv was accordingly reduced, and determined CL1 to present a variation below $0.1 \%$ for the majority of Qins, as seen in Figure 10 (b), therefore establishing a more uniform environment.

Following a similar purpose, the shape of the main chamber's outlet was changed from a single channel, acknowledged to provide a steep variation towards the end of the chamber, as visible in Figure 11 (a), to an array of channels, meant to collect flow streams without disrupting the 02 concentration distribution. Overall, these modifications ensured that along each CL, $\mathrm{O} 2$ concentration levels were kept below $0.5 \%$ of variation, as demonstrated through Figure 11 (b).
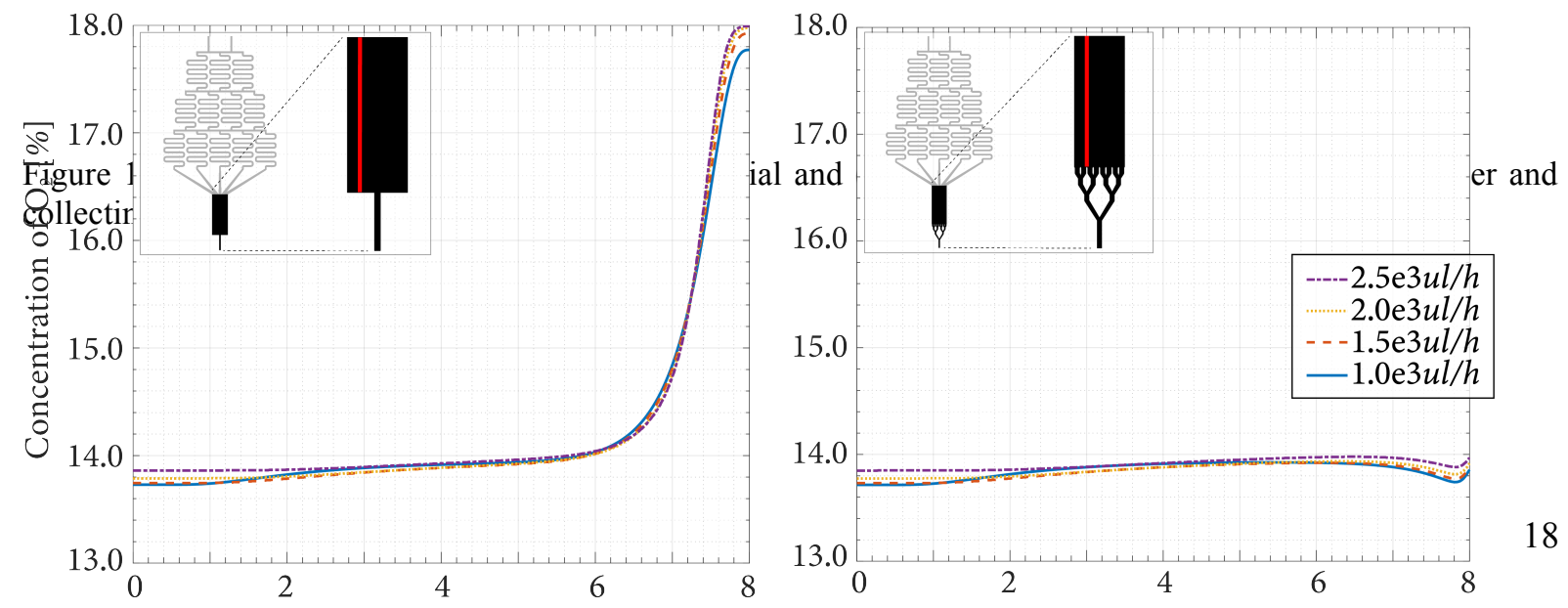

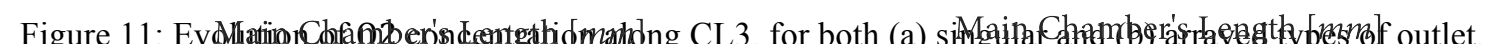




\subsection{Height of Channels}

The height of channels, as denoted previously by Table 4, was acknowledged to provide crucial control over shear stress, and its effect was studied by employing a 3D model, achieved by extruding the previous 2D model of the device in present analysis. Shear stress was studied at the main chamber and analysed along the surface defined by the height of the liver organoids. Due to the heterogeneous cell composition of organoids, shear stress must be adjusted in accordance with the various liver cell types composing the respective structures.

Literature is clear to indicate the importance of regulating shear stress towards ensuring a correct physiological behaviour of cells $[39,41,42]$. Regarding liver tissue particularly, literature indicates that a moderate shear stress must be employed to ensure appropriate culture, morphology and function [35-37]. However, optimal values for shear stress are reported with a considerable variance within literature examples. Ledezma et al. [39] addresses a threshold shear stress of $10 \mathrm{dyn} / \mathrm{cm} 2(=1 \mathrm{~Pa})$ as being conservative to liver hepatocyte viability, which fits within the moderate shear stress intervals as suggested by Song et al. [36] and Torii et al. [37], to respectively ensure correct morphology of endothelial cells, for an interval of $5 \mathrm{dyn} / \mathrm{cm} 2(=0.5 \mathrm{~Pa})$ to $20 \mathrm{dyn} / \mathrm{cm} 2(=2 \mathrm{~Pa}$ ), and maintain liver specific functions, for a range of $0.5 \mathrm{dyn} / \mathrm{cm} 2(=0.05 \mathrm{~Pa})$ to $20 \mathrm{dyn} / \mathrm{cm} 2(=2 \mathrm{~Pa})$. Tanaka et al. [35] reports that, within a normal hepatic sinusoid, shear stress is as high as $0.5 \mathrm{~Pa}$, whereas Rashidi et al. [38] further specifies the optimal shear stress interval to a range between $0.1 \mathrm{dm} 2(=0.01 \mathrm{~Pa})$ and 0.5 $d y n / c m 2(=0.05 \mathrm{~Pa})$.

Being the most restrictive, the interval referenced by Rashidi et al. [38] was determined as the ideal shear stress range to ensure liver tissue culture, morphology and function of greater physiological relevance. The height of channels was refined accordingly, to ultimately assume the value of $0.40 \mathrm{~mm}$, for which numerical simulations predicted shear stress to be comprised within the determined interval, for the respective Qin range in analysis. Simulation results are expressed in Table 5, whereas Figure 12 illustrates an average surface shear stress of $0.037 \mathrm{~Pa}$ obtained for $\mathrm{Qin}=$ with channels of height

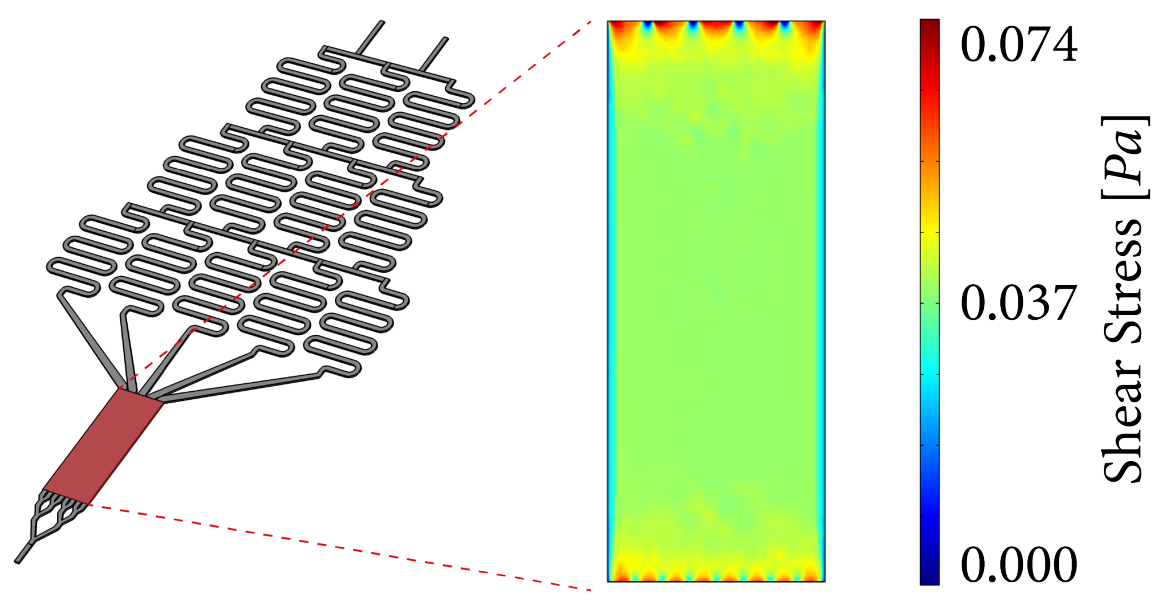


equal to $0.40 \mathrm{~mm}$.

\section{TABLE 5}

\subsection{Final Iteration for Inlet Flow Rate}

With the geometry fully defined, Qin was ultimately determined to a single value, thus fully defining the ideal solution to enhance 02 gradient generation within the $\mathrm{GoaC}$ device. The selection procedure to find the most suitable Qin was carried by considering the previously established data, as well as establishing new arguments to sustain the selected value of $Q$ in.

Given the narrow range of the Qin interval in present analysis, changes between the microenvironments generated by applying either one or the other end of the Qin spectrum, will be small and very specific, but nonetheless, relevant. Numerical simulations suggested their respective influence to mainly control gradient stability, along each $\mathrm{CL}$, and profile smoothness, across each GL. It was determined that, for a lower Qin, the gradient would reach a smoother, and therefore more desirable profile sooner, within the main chamber, as seen by the blue continuous line in Figure 13, even if in detriment of less stable CLs, which characterizes a gradient more prone to fade. On the other hand, employing a higher Qin meant that 02 would have less time to diffuse, further characterizing the gradient as having a less continuous profile across GLs, visible by the purple discontinuous line in Figure 13, but rather defining steadier values along each CL, suggesting a more uniform 02 concentration environment. The most suitable value was found in between, for $Q$ in $=1.5 \times 103 \mu L / h$, to provide a balance between a smoother gradient and a more uniform 02 concentration environment along the entire main chamber, as visible in Figure 14. By selecting the referred value, it was also possible to provide highly uniform 02 concentration levels after each serpentine channel, with a precision of approximately $0.1 \%$ (visible in Figure 8 (b)), maintain each CL along the main chamber below a variance of $0.5 \%$ of 02 concentration (Figure $10(\mathrm{~b})$ ), and determine a shear stress distribution
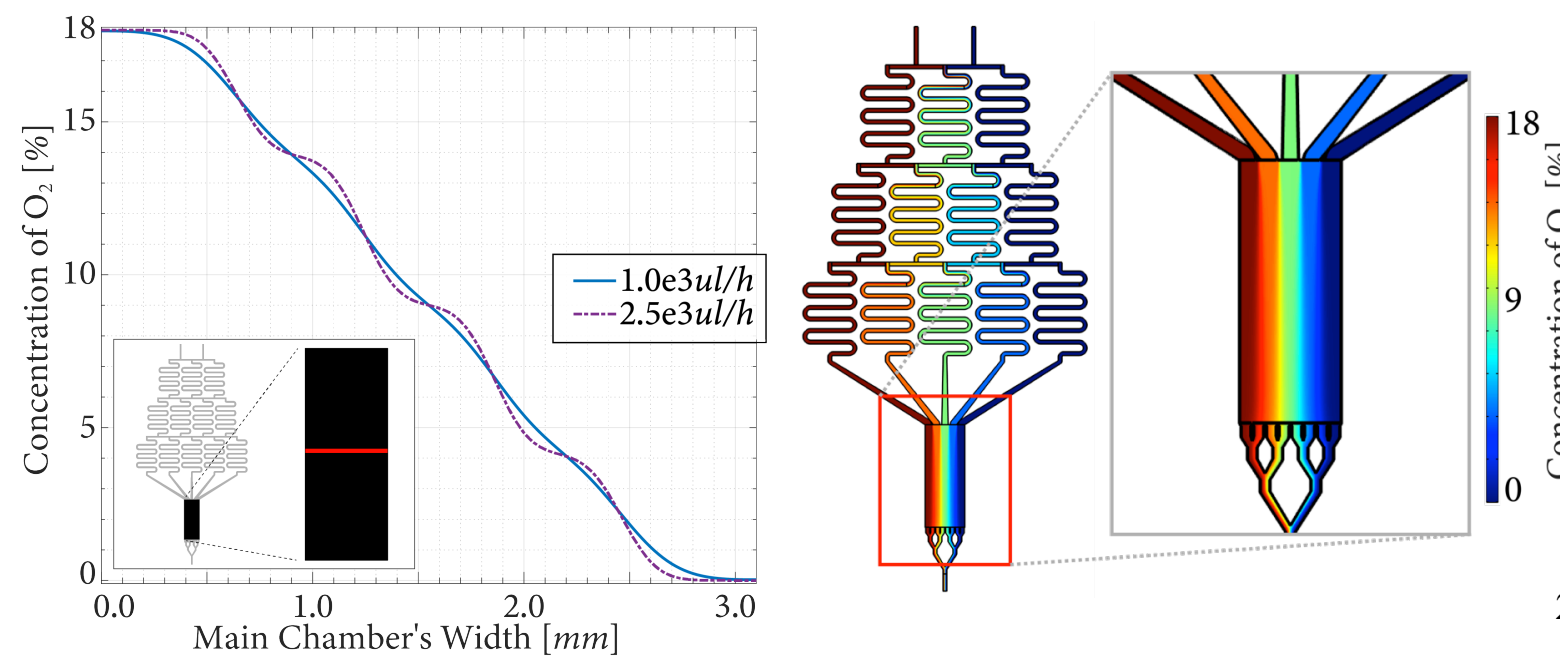
with an average magnitude of $0.037 \mathrm{~Pa}$ (as established through Table 5), well within the interval defined as most appropriate, to therefore ensure physiologically relevant culture, morphology and function of liver organoids.

Therefore, through the present solution, the 02 concentration gradient was refined to be distributed along the main chamber in a highly uniform and deterministic manner, by establishing continuous CLs, as seen in Figure 15 (a), and smooth gradient profiles along GLs. However, the human liver relevant 02 zonation, known to be comprised between $10-12 \%$, for the periportal, and $3-5 \%$, for the perivenous zones [14], is present at the main chamber of the developed device within only $40.6 \%$ of the respective domain, as seen through the GLs in Figure 15 (b) where the red coloured area A represents the periportal zone, $\mathrm{B}$ the transitional zone, and the blue coloured area $\mathrm{C}$ the perivenous

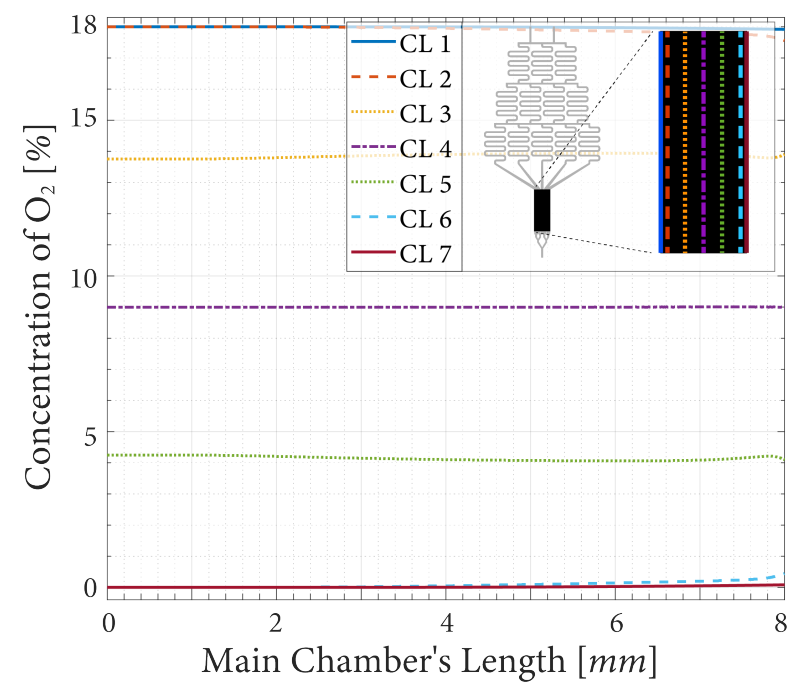

zone.

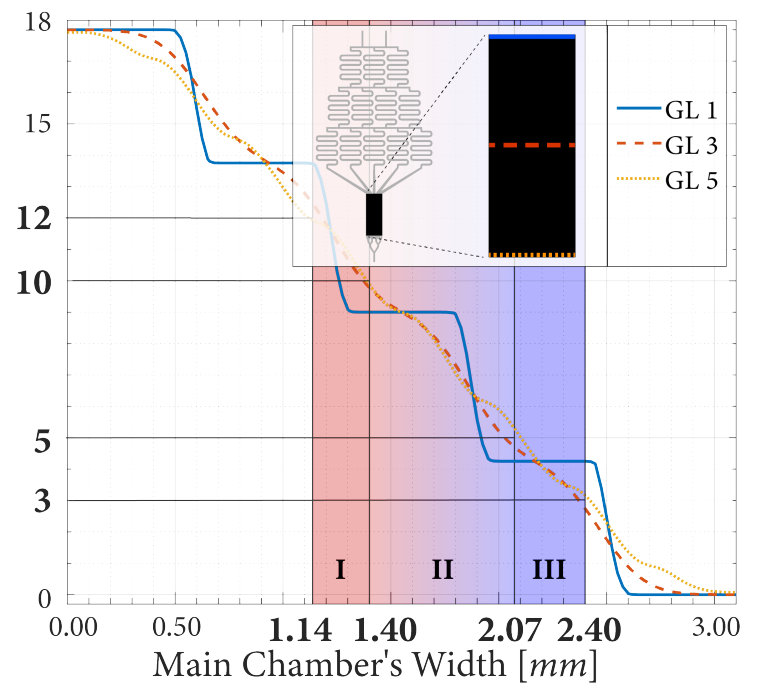

(b)

\subsection{The Concentration Regulator Module}

By improving the geometry and fluidic parameters defining the GoaC device, it was possible to refine and perfect $\mathrm{O} 2$ gradient generation. However, to create an improved environment specifically meant for liver modelling, the necessity of controlling gradient generation emerged, towards readjusting 02 values to be comprised within a range of greater relevance and accuracy in regard to the acinus in vivo zonation. Therefore, and by taking advantage of both parametric and modular capabilities of the developed finite element framework, a structure of novel functionality was developed, demonstrated in Figure 16 and designated as concentration regulator (CR) module, enabling full control over gradient generation, and requiring only two input concentration values. Following a similar serpentine channel Figure 15: Oxygen gradient distribution across (a) every CL and (b) GL1, 3 and 5, where the red-colored area "A" represents the periportal zone within the main chamber, "B" the transitional zone and the blue-colored area "C" comprises the perivenous zone.

structure for mixing, the CR further enhances the respective structure's purpose to, not only split, mix and therefore expand the concentration differential established at the inlets, but also converge, remix 




Figure 16: Concentration regulator module illustrating both diverging and converging steps.

and narrow the same respective interval. Such feature is accomplished by a diamond shaped design consisting of one row of inlet channels and two of serpentines, to define a diverging step immediately followed by a converging one. Ultimately, the present design achieves full control over the output concentration interval which, if coupled to the $\mathrm{GoaC}$, enables complete modulation of gradient generation, by either shifting or regulating its range, through control of two ratios regarding flow rate and hydraulic resistance. Changing the inlet flow rate ratio, RQin, enables shifting the gradient, as demonstrated through Figure 17 (d), to either ends of the concentration interval established at the inlets. The ratio can be defined as follows:

RQin=QinLeft

(8)

\section{ChannelQinRight}

\section{Channel}

If RQin=1 (Figure 17 (b)), it determines that Qin is equal in magnitude for both inlet channels. Due to the converging step, the gradient will display as lower and upper boundary, the first and third quarters of the concentration differential established at the inlets, respectively. However, if the ratio is changed to RQin $\neq 1$, it means that flow will either favour the concentration saturated inlet, determining a higher ranged gradient, for RQin>1 (Figure 17 (a)), or the concentration depleted inlet, establishing a lower ranged gradient, for RQin<1, (Figure 17 (c)).

Adding to gradient shifting, correctly determining the hydraulic resistance ratio, Rhyd between outer and middle serpentine channels, at the second row of the $\mathrm{CR}$, enables control over the range presented by the gradient, graphically denoted by the change in steepness, as demonstrated by Figure 18 (d). It is first required to define hydraulic resistance, $R h y d$, which for channels with a rectangular cross-section is expressed as follows [25]:

$R h y d=12 \mu l 1-0.63(h / w) 1 h 3 w$ 
where $l$ is the length of the channel, $w$ its width and $h$ its height. Through the present analysis, the parameter used for manipulation of Rhyd was the width of channels. It is then possible to define $\mathrm{R} h y d$ as being:

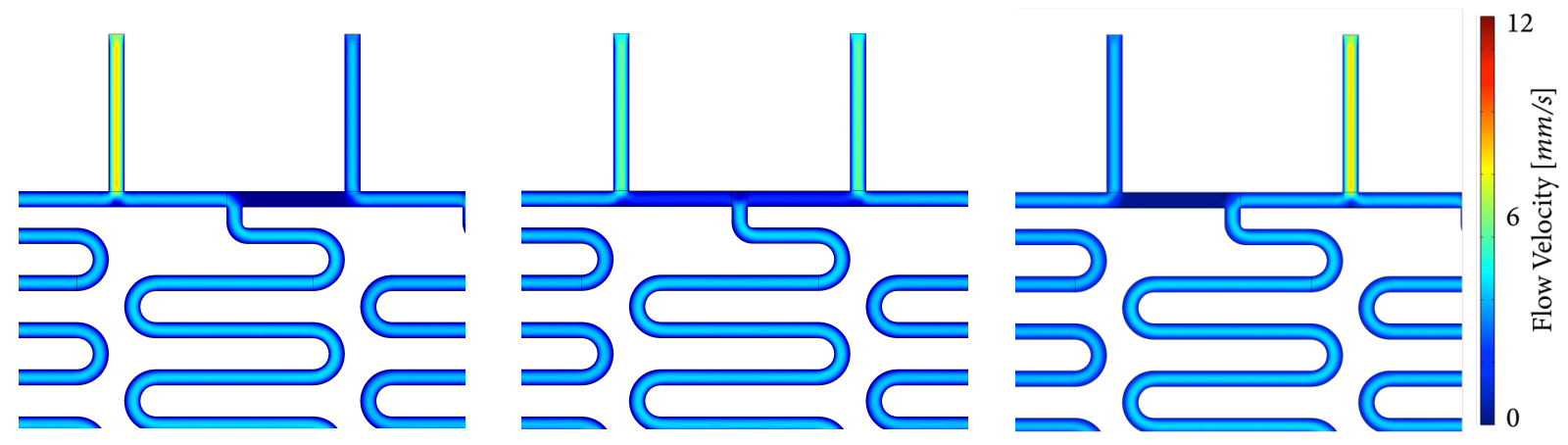



(10) (b)

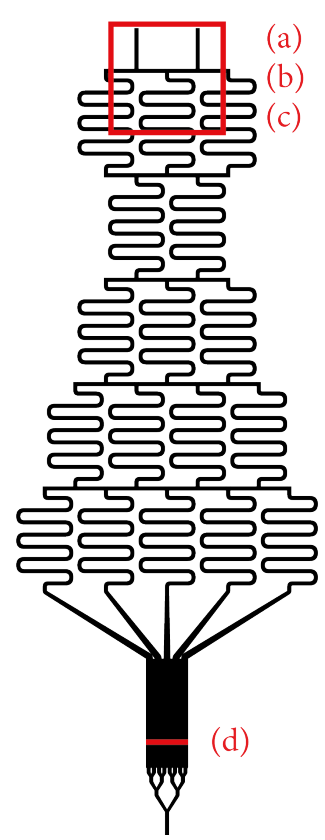

(c)

(d)

If Rhyd>1 (Figure 18 (a)), it means the RhydMiddle Serpentine is higher, and therefore, less flow will cross the respective channel, further hindering mixing at the divergent step and ultimately resulting in a steeper gradient of wider range. However, if the opposite condition is met, Rhyd<1 (Figure 18 (c)), it determines a lower RhydMiddle Serpentine, which therefore will enhance flow passage at the respective channel. Such phenomena will enhance mixing, ultimately generating a gradient with values closer to the concentration equilibrium state.

Figure 17: (a) $\mathrm{R} \quad>1$, corresponding to the blue dashed line in (d). (b) $\mathrm{R} \quad=1$, corresponding to the yellow continuous line in (d). (c) $\mathrm{R} \quad<1$, corresponding to the red dotted dashed line in (d). (d) 02 concentration gradient shifting enabled by controlling the $\mathrm{R}$ through the CR module. (e) whole design evidencing the domains in analysis by figures (a), (b) and (c), as well as (d). 
If this module is carefully adjusted in regard to human liver zonation, by shifting and narrowing the 02 gradient produced through manipulation of the ratios presented, it is possible to obtain a good correlation with the 02 gradient values as found in vivo within the liver acinus and along the sinusoid. This is obtained by defining the ratios as being RQin=0.71, representing a shifting of the gradient towards the lower 02 concentration range of the differential established at the inlets, and $\mathrm{R} h y d=0.21$, defining a narrower gradient to precisely replicate human liver acinus 02 concentration values, limited
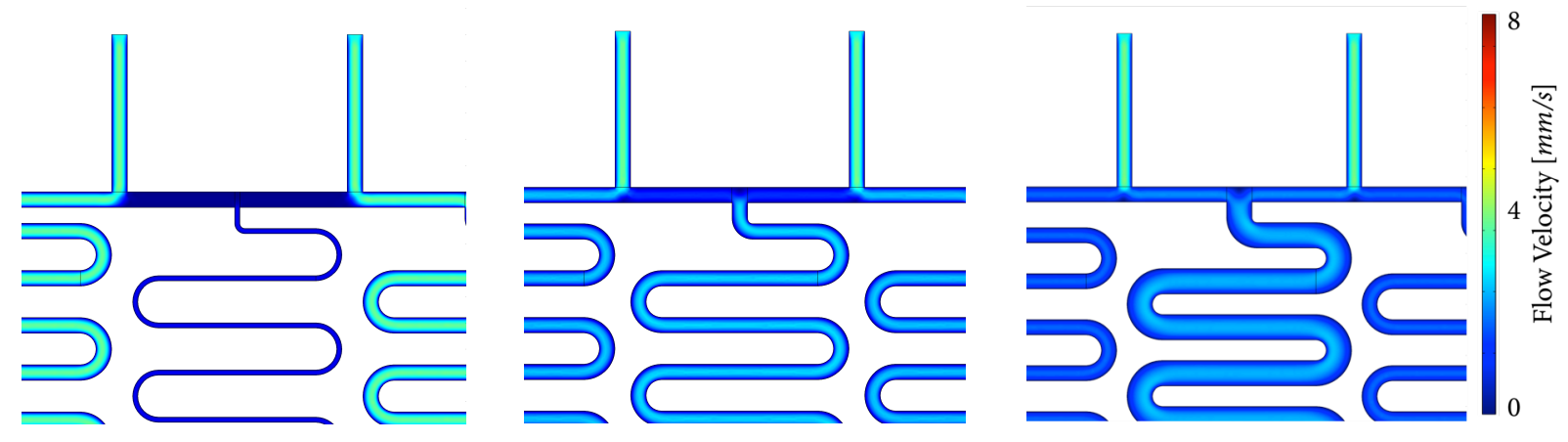

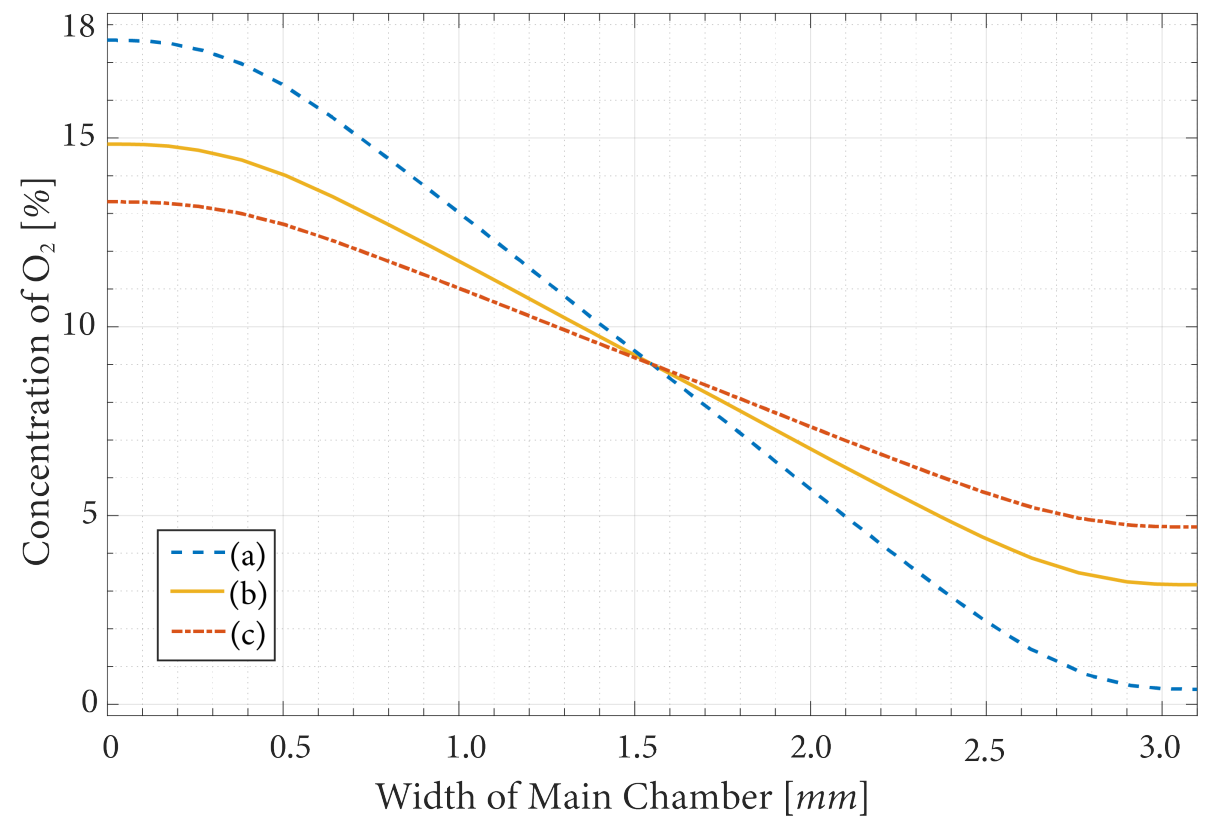

(a) (b)

(d)

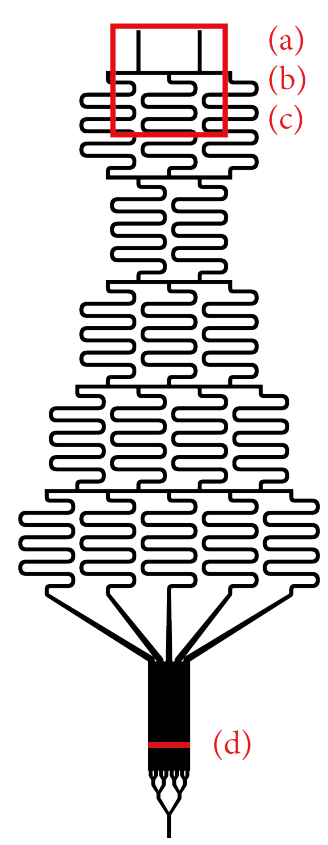

(c)

(e)

above by the periportal zone, and below by the perivenous zone. Such configuration enabled the liver relevant gradient of 02 to be extended across the whole of the main chamber's domain, as visible in Figure 19. Furthermore, all the previously determined properties, determined to ensure a smooth gradient of uniform distribution along the entire main chamber, were acknowledged to remain unchanged, as demonstrated through the steady profiles of the CLs in Figure 20. The present device is designated as liver gradient-on-a-chip, LGoaC, and it represents the most appropriate solution to generate in vitro gradients of 02 as found in vivo within the human liver acinus, therefore standing as a more accurate and relevant liver model for OoaC applications. 


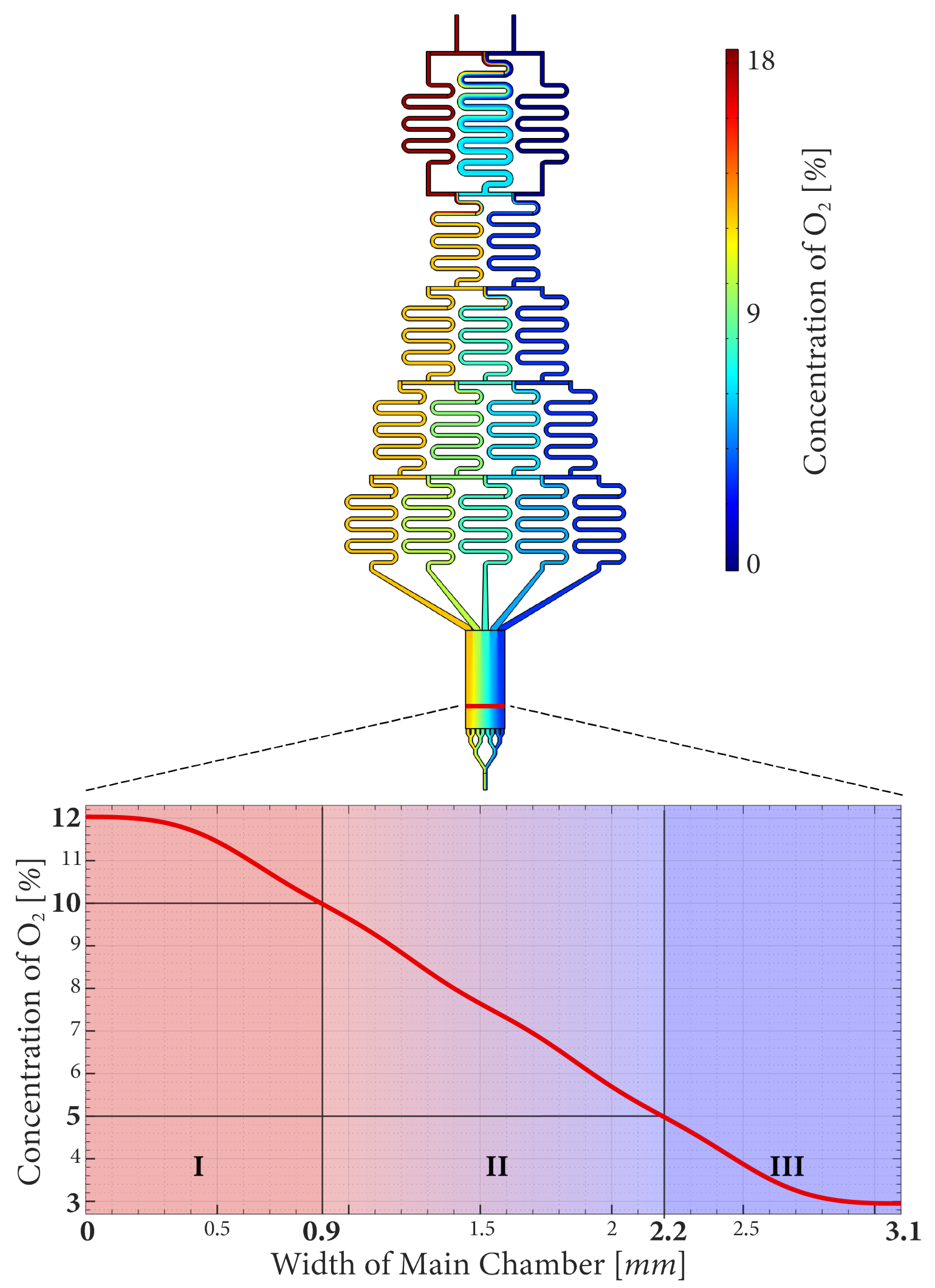




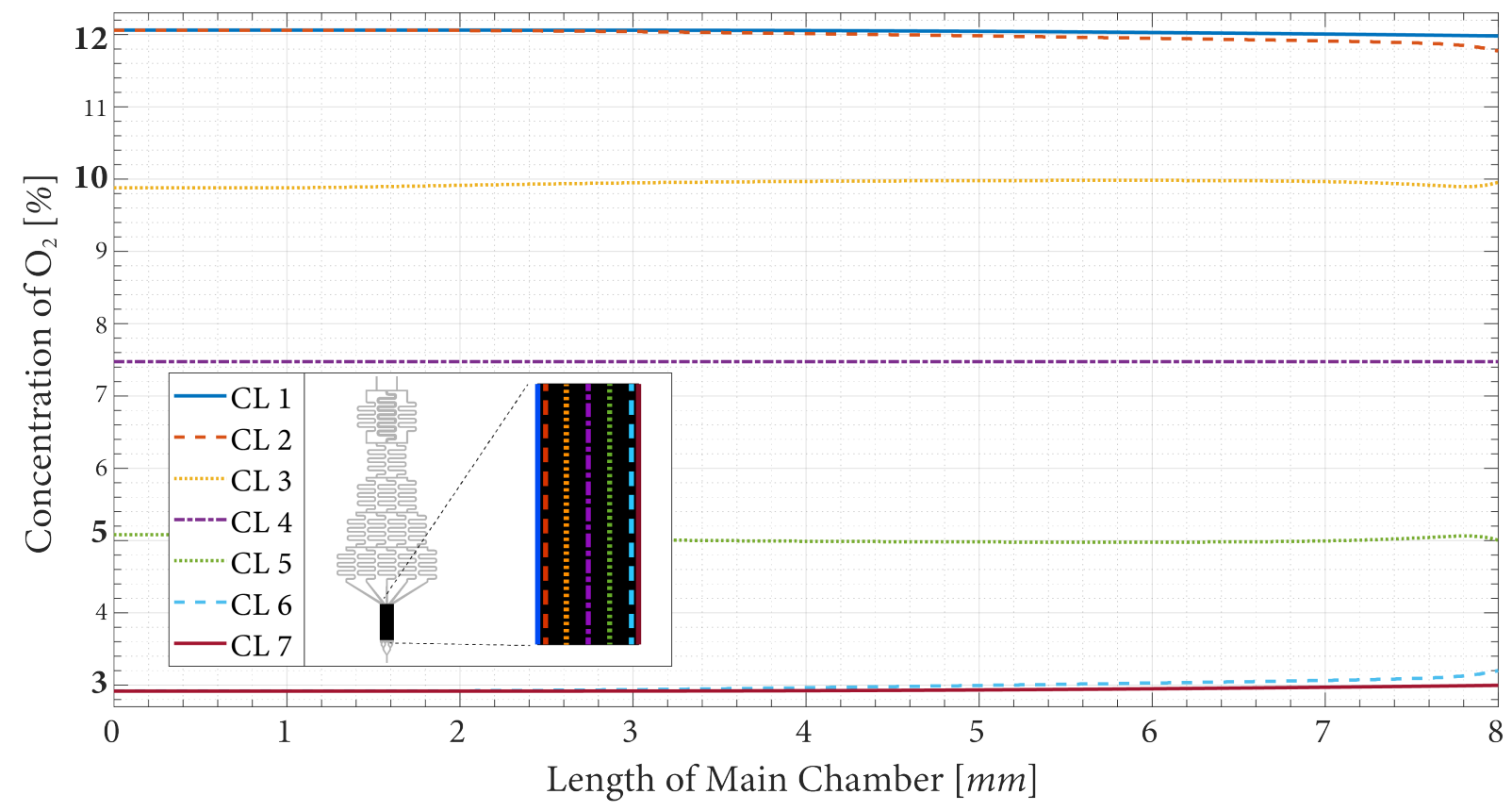

Figure 20: Evolution of $\mathrm{O} 2$ concentration levels along every CL.

\subsection{Liver Gradient-on-a-chip, the Ideal Solution}

Table 6 presents the list of parameters which collectively define the solution found as most appropriate for in vitro generation of 02 gradients as found in vivo within the human liver acinus. Parameters are further illustrated by Figure 21 to fully define the obtained liver model.

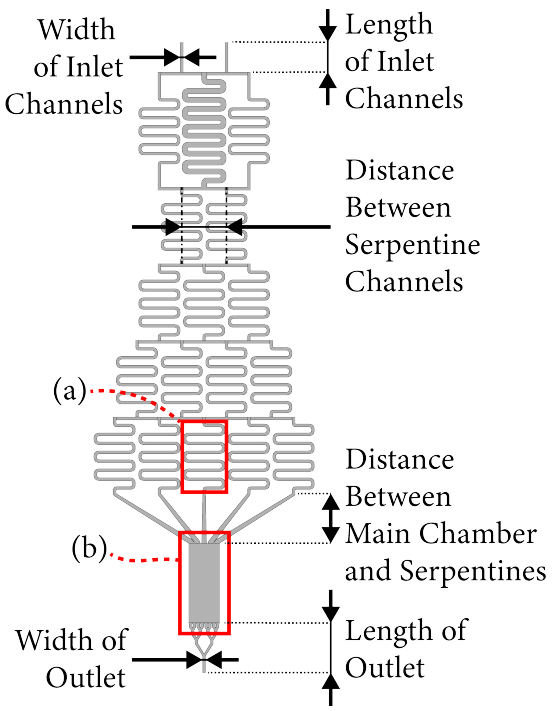

(a)

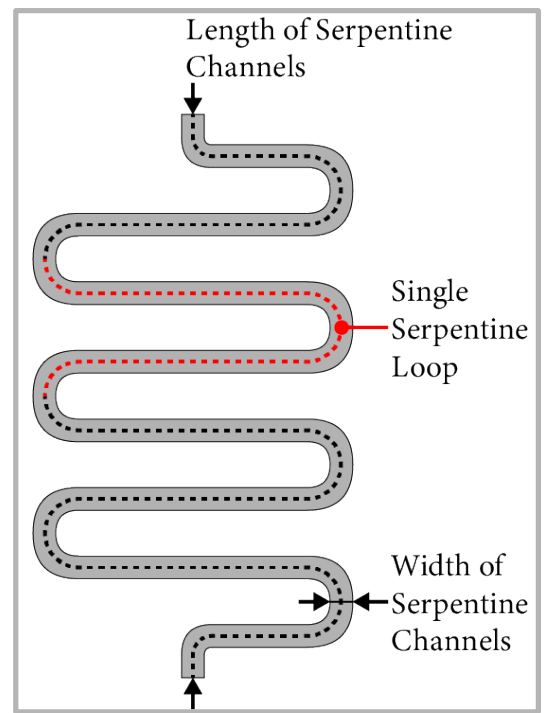

(b)

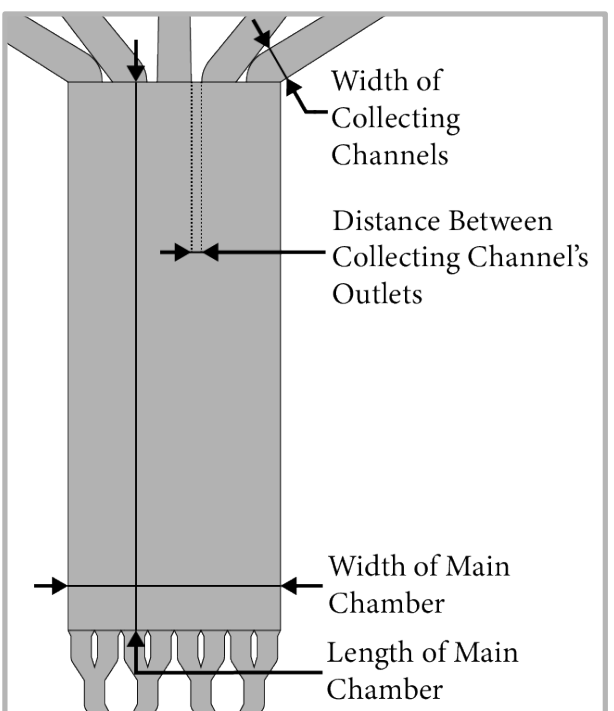

(c)

TABLE 6 


\section{Conclusion}

The present work focused on developing a capable and relevant methodology to develop a gradient generator, designated as Gradient-on-a-chip ( $\mathrm{GoaC}$ ), and produce deterministic gradients of 02 uniformly spread across the cell culturing site, as well as relevant shear stress values, to more precisely recapitulate, in vitro, the human liver microphysiology, as found in vivo.

The developed finite element framework was based on COMSOL Multyphysics ${ }^{\circledR}$ modelling software to simulate the underlying physics describing OoaC applications. Furthermore, it was established a language based on the software's interface to create a model that is both parametric and modular. This approach required the development of parameter-based relations, to maintain geometric coherence within the device for every intended combination and modular entity. The present methodology enabled the study of how hydrodynamics can be modelled by geometry to create more specific and purposeful devices. By successfully replicating two distinct experimental applications from literature, the present approach was provided with robust arguments to sustain its own legitimacy on simulating both gradient generation and flow-induced shear stress, fundamental features for the present study.

The established finite element approach was employed to refine the $\mathrm{GoaC}$ towards generating human liver relevant gradients of 02 . Initially, parameters were individually evaluated, from which it was possible to determine and rank their own respective influence, and establish a design refinement procedure based on the obtained data. It was proceeded with the refinement of the $\mathrm{GoaC}$, having as a goal the enhancement of the gradient of 02 established at the main chamber, towards defining a deterministic and uniform environment, characterized by smooth gradient profiles, across each GL, and steady concentration levels, managing to maintain a variation below $0.5 \%$ along each CL. Shear stress was also modulated to be equal to $0.037 \mathrm{~Pa}$ and meet the values determined from literature to enhance liver tissue culture, morphology and function. Finally, with the purpose of obtaining complete control over gradient generation, it was developed a structure of novel functionality, designated as concentration regulator (CR), from which it was possible to either shift or regulate the range of the concentration gradient, towards meeting the specific requirements of liver zonation. Coupled with the $\mathrm{GoaC}$, the $\mathrm{CR}$ was further adjusted to obtain a collective solution of improved functionality, designated as liver gradient-on-a-chip (LGoaC), capable of producing gradients ranging from $3 \%$ to $12 \%$, and therefore providing a precise correlation with the values found in vivo within the human liver acinus, reported to be comprised between the perivenous, $3-5 \%$ of 02 , and the periportal zones, $10-12 \%$ of 02 .

Given the device's specificity towards establishing a microenvironment of greater physiological pertinence for culture and growth of liver organoids, by defining appropriate levels of shear stress and determining gradients of $\mathrm{O} 2$ that precisely replicate the values found in vivo within the human liver acinus, the LGoaC is believed to stand as a liver model of greater accuracy and relevance for OoaC applications. 
Due to its foreseen value, the concentration regulator module could be object of greater development towards providing improved flexibility and functionality over gradient generation during experimental testing. The current proposed solution only envisions flow rate ratio as a parameter capable of being changed during live experimental testing, thus solely enabling real-time shifting of the developed gradient, which is one of the limitations of the study.

The final solution for the liver gradient-on-a-chip was further characterized by specifying each respective parameter, towards enabling other researchers to readily reproduce experimentally the device here reported.

Beyond the developed solution, it is equally highlighted the importance of the employed design procedure, which should be re-iterated if considering the simulation of a distinct concentration gradient and microenvironment, for which the different flowing media and diluted particle conditions, will demand respectively for a change in the design settings of the device.

\section{Acknowledgments}

The authors gratefully acknowledge support by the Research Council of Norway through its Centres of Excellence funding scheme, project number 262613. Moreover, the authors acknowledge support by the James Watt School of University of Glasgow, the Engineering Faculty of University of Porto (FEUP), the Institute of Science and Innovation in Mechanical and Industrial Engineering (INEGI), and all the staff, for supporting and helping with the making of the present project.

\section{Abbreviations}

CFD

CL

$\mathrm{CR}$

GL

GoaC

LGoaC

$\mathrm{OoaC}$

$2 \mathrm{D}$

$3 \mathrm{D}$
Computational fluid dynamics

Concentration line

Concentration regulator

Gradient line

Gradient-on-a-chip

Liver gradient-on-a-chip

Organ-on-a-chip

Two dimensional

Three dimensional

\section{Notation}

$\nabla$

C

D

F
Upper-convected derivative

Concentration $[\mathrm{mol} / \mathrm{m} 3]$

Diffusion coefficient rate $[\mathrm{m} 2 / \mathrm{s}]$

Volume force per unit mass $[\mathrm{N} / \mathrm{kg}]$ 
$P e$

Qin

$\mathrm{R}$

Re

Rhyd

$S$

SAStPtX

SAdboc

SAdbs

SAwoc

$S w c$

$t$

$U$

$u$

Uin

$w$

\section{Greek Symbols}

$\mu$

$\rho$

\section{Chemical Formulas}

O2
Identity matrix

Height $[m]$

Length $[m]$

Number of flow streams

Pressure $[\mathrm{Pa}]$

Péclet number

Inlet flow rate $[\mathrm{m} 3 / \mathrm{s}]$

Ratio

Reynolds number

Hydraulic resistance $[\mathrm{kg} /(\mathrm{m} 4 \mathrm{~s})]$

Source/ sink of mass $[\mathrm{mol} /(\mathrm{m} 2 \mathrm{~s})]$

Distance of upstream mixer along $\mathrm{x}$ axis $[\mathrm{mm}]$

Distance between outlet of collecting channels $[\mathrm{mm}]$

Distance between serpentines [mm]

Width of outlet of collecting channels [mm]

Width of serpentine channels $[\mathrm{mm}]$

Time $[s]$

Mean velocity $[\mathrm{m} / \mathrm{s}]$

Velocity $[\mathrm{m} / \mathrm{s}]$

Inlet flow velocity $[\mathrm{m} / \mathrm{s}]$

Width $[m]$

Density $[\mathrm{kg} / \mathrm{m} 3]$

Oxygen 


\section{References}

1. Harrison RG. The outgrowth of the nerve fiber as a mode of protoplasmic movement. Journal of Experimental Zoology 1910; 9(4):787-846. doi:10.1002/jez.1400090405.

2. Ronaldson-Bouchard K, Vunjak-Novakovic G. Organs-on-a-Chip: A Fast Track for Engineered Human Tissues in Drug Development. Cell Stem Cell 2018; 22(3):310-324. doi:10.1016/j.stem.2018.02.011.

3. Ehrmann RL, Gey GO. The Growth of Cells on a Transparent Gel of Reconstituted Rat-Tail Collagen2. JNCI: Journal of the National Cancer Institute 1956; 16(6):1375-1403. doi:10.1093/jnci/16.6.1375.

4. Park TH, Shuler ML. Integration of cell culture and microfabrication technology. Biotechnology Progress 2003; 19(2):243-253. doi:10.1021/bp020143k.

5. Bhatia SN, Ingber DE. Microfluidic organs-on-chips. Nature Biotechnology 2014; 32(8):760772. doi:10.1038/nbt.2989.

6. Loskill P, Sezhian T, Tharp KM, et al. WAT-on-a-chip: A physiologically relevant microfluidic system incorporating white adipose tissue. Lab on a Chip 2017; 17(9):1645-1654. doi:10.1039/c6lc01590e.

7. Ahadian S, Civitarese R, Bannerman D, et al. Organ-On-A-Chip Platforms: A Convergence of Advanced Materials, Cells, and Microscale Technologies. Advanced Healthcare Materials 2018; 7(2). doi:10.1002/adhm.201700506.

8. Plenge RM. Disciplined approach to drug discovery and early development. Science Translational Medicine 2016; 8(349):1-6. doi:10.1126/scitranslmed.aaf2608.

9. Capulli AK, Tian K, Mehandru N, et al. Approaching the in vitro clinical trial: Engineering organs on chips. Lab on a Chip 2014; 14(17):3181-3186. doi:10.1039/c4lc00276h.

10. Franzen N, van Harten WH, Retèl VP, Loskill P, van den Eijnden-van Raaij J, IJzerman M. Impact of organ-on-a-chip technology on pharmaceutical R\&D costs. Drug Discovery Today 2019; 24(9):1720-1724. doi:10.1016/j.drudis.2019.06.003.

11. Tomlinson L, Hyndman L, Firman JW, et al. In vitro Liver Zonation of Primary Rat Hepatocytes. Frontiers in Bioengineering and Biotechnology 2019; 7(February):1-8. doi:10.3389/fbioe.2019.00017.

12. Kietzmann T. Metabolic zonation of the liver: The oxygen gradient revisited. Redox Biology 2017; 11(December 2016):622-630. doi:10.1016/j.redox.2017.01.012.

13. Godoy $\mathrm{P}$, Hewitt NJ, Albrecht $\mathrm{U}$, et al. Recent advances in $2 D$ and $3 D$ in vitro systems using primary hepatocytes, alternative hepatocyte sources and non-parenchymal liver cells and their use in investigating mechanisms of hepatotoxicity, cell signaling and ADME.; 2013.

14. Lee-Montiel FT, George SM, Gough AH, et al. Control of oxygen tension recapitulates zonespecific functions in human liver microphysiology systems. Experimental Biology and Medicine 2017; 242(16):1617-1632. doi:10.1177/1535370217703978. 
15. Moradi E, Jalili-Firoozinezhad S, Solati-Hashjin M. Microfluidic organ-on-a-chip models of human liver tissue. Acta Biomaterialia 2020; 116:67-83. doi:10.1016/j.actbio.2020.08.041.

16. Convery N, Gadegaard N. 30 Years of Microfluidics. Micro and Nano Engineering 2019; 2(November 2018):76-91. doi:10.1016/j.mne.2019.01.003.

17. Kannan R, Chen ZJ, Singh N, et al. A quasi-3D wire approach to model pulmonary airflow in human airways. International Journal for Numerical Methods in Biomedical Engineering 2017; 33(7):1-13. doi:10.1002/cnm.2838.

18. Lee TR, Hong JA, Yoo SS, Kim DW. A computational modeling of blood flow in asymmetrically bifurcating microvessels and its experimental validation. International Journal for Numerical Methods in Biomedical Engineering 2018; 34(6):1-19. doi:10.1002/cnm.2981.

19. Marturano-Kruik A, Villasante A, Yaeger K, et al. Model of Human Tumor. Biomaterials 2018; 150:150-161. doi:10.1016/j.biomaterials.2017.10.020.Biomechanical.

20. Achberger K, Probst C, Haderspeck JC, et al. Merging organoid and organ-on-a-chip technology to generate complex multi-layer tissue models in a human retina-on-a-chip platform. eLife 2019; 8. doi:10.7554/eLife.46188.

21. Tabeling P. Introduction to microlfuidics. 2005:312.

22. Jeon NL, Dertinger SKW, Chiu DT, Choi IS, Stroock AD, Whitesides GM. Generation of solution and surface gradients using microfluidic systems. Langmuir 2000; 16(22):8311-8316. doi:10.1021/la000600b.

23. Keenan TM, Folch A. Biomolecular gradients in cell culture systems. Lab on a Chip 2007; 8(1):34-57. doi:10.1039/b711887b.

24. Park SE, Georgescu A, Huh D. O r ga noid s review. 2019; 965(June):960-965.

25. Bruus H. Theoretical microfluidics. Choice Reviews Online 2008; 45(10):45-5602-45-5602. doi:10.5860/choice.45-5602.

26. Nguyen NT, Shaegh SAM, Kashaninejad N, Phan DT. Design, fabrication and characterization of drug delivery systems based on lab-on-a-chip technology. Advanced Drug Delivery Reviews 2013; 65(11-12):1403-1419. doi:10.1016/j.addr.2013.05.008.

27. Li Jeon N, Baskaran H, Dertinger SKW, Whitesides GM, De Water L Van, Toner M. Neutrophil chemotaxis in linear and complex gradients of interleukin-8 formed in a microfabricated device. Nature Biotechnology 2002; 20(8):826-830. doi:10.1038/nbt712.

28. Okiishi M, Rothmayer H. Fundamentals of Fluid Mechanics.

29. Beebe DJ, Mensing GA, Walker GM. Physics and Applications of Microfluidics in Biology. Annual Review of Biomedical Engineering 2002; 4(1):261-286. doi:10.1146/annurev.bioeng.4.112601.125916.

30. Batchelor GK. An Introduction to Fluid Dynamics. Physics Today 1959; 12(4):36-38. doi:10.1063/1.3060769.

31. Einstein A, Ph D. -i nvestigations o $\mathrm{n}$ the. 1956.

32. Lauga E, Brenner M, Stone H. Microfluidics: The no-slip boundary condition. Springer 
Handbooks 2007:1219-1240. doi:10.1007/978-3-540-30299-5_19.

33. Newby D, Marks L, Lyall F. Dissolved oxygen concentration in culture medium: Assumptions and pitfalls. Placenta 2005; 26(4):353-357. doi:10.1016/j.placenta.2004.07.002.

34. Ochs CJ, Kasuya J, Pavesi A, Kamm RD. Oxygen levels in thermoplastic microfluidic devices during cell culture. Lab on a chip 2014; 14(3):459-462. doi:10.1039/c3lc51160j.

35. Tanaka Y, Yamato M, Okano T, Kitamori T, Sato K. Evaluation of effects of shear stress on hepatocytes by a microchip-based system. Measurement Science and Technology 2006; 17(12):3167-3170. doi:10.1088/0957-0233/17/12/S08.

36. Song JW, Gu W, Futai N, Warner KA, Nor JE, Takayama S. Computer-controlled microcirculatory support system for endothelial cell culture and shearing. Analytical Chemistry 2005; 77(13):3993-3999. doi:10.1021/ac050131o.

37. Torii T, Miyazawa M, Koyama I. Effect of continuous application of shear stress on liver tissue: Continuous application of appropriate shear stress has advantage in protection of liver tissue. Transplantation Proceedings 2005; 37(10):4575-4578. doi:10.1016/j.transproceed.2005.10.118.

38. Rashidi H, Alhaque S, Szkolnicka D, Flint O, Hay DC. Fluid shear stress modulation of hepatocyte-like cell function. Archives of Toxicology 2016; 90(7):1757-1761. doi:10.1007/s00204-016-1689-8.

39. Ledezma GA, Folch A, Bhatia SN, Balis UJ, Yarmush ML, Toner M. Numerical model of fluid flow and oxygen transport in a radial-flow microchannel containing hepatocytes. Journal of Biomechanical Engineering 1999; 121(1):58-64. doi:10.1115/1.2798043.

40. Cooksey GA, Sip CG, Folch A. A multi-purpose microfluidic perfusion system with combinatorial choice of inputs, mixtures, gradient patterns, and flow rates. Lab on a Chip 2009; 9(3):417-426. doi:10.1039/b806803h.

41. Mazza G, Al-Akkad W, Telese A, et al. Rapid production of human liver scaffolds for functional tissue engineering by high shear stress oscillation-decellularization. Scientific Reports 2017; 7(1):1-14. doi:10.1038/s41598-017-05134-1.

42. Stone SD, Hollins BC. Modeling shear stress in microfluidic channels for cellular applications. Proceedings - 29th Southern Biomedical Engineering Conference, SBEC 2013 2013; (May):117-118. doi:10.1109/SBEC.2013.67.

43. Kundu PK, Cohen IM, Dowling DR eds. Appendix A - Conversion Factors, Constants, and Fluid Properties. In: Fluid Mechanics (Sixth Edition), Sixth Edit. Boston: Academic Press; 2016:881-884.

44. Novotný P, Söhnel O. Densities of Binary Aqueous Solutions of 306 Inorganic Substances. Journal of Chemical and Engineering Data 1988; 33(1):49-55. doi:10.1021/je00051a018. 


\section{Tables}

Table 1: Parameters defining testing conditions, medium and dissolved species.

\begin{tabular}{|c|c|c|}
\hline Parameter & Value & Reference \\
\hline Testing Temperature, $[\underline{\underline{o}}]$ & 37 & - \\
\hline Density of Water at $37\left[^{o}\right],[/ 3]$ & 993.30 & [43] \\
\hline Dynamic Viscosity of Water at $37\left[^{o}\right],[\cdot]$ & 0.6965 & [43] \\
\hline Diffusion Coefficient of $\mathbf{0 2}$ in Water at $37\left[^{o}\right],[2 /]$ & $3.35 \times 10-9$ & [34] \\
\hline Concentration Supply of $\mathbf{0 2}$ in Water at $37\left[^{o}\right],[/ 3]$ & 0.17075 & {$[33,34]$} \\
\hline
\end{tabular}

Table 2: Parameters defining medium and dissolved species for Example 1.

\begin{tabular}{lcc}
\hline Parameter & Value & Reference \\
\hline Density of Sodium Bicarbonate, $[/ 3]$ & 1023.1 & {$[44]$} \\
\hline Dynamic Viscosity of Sodium Bicarbonate, $[\cdot]$ & 0.0011020 & {$[44]$} \\
\hline Diffusion Coefficient of Fluorescein Isothiocyanate, $[2 /]$ & $5 \times 10-10$ & {$[22]$} \\
\hline Concentration Supply of Fluorescein Isothiocyanate, $[\mathbf{~}]$ & 100 & {$[22]$} \\
\hline
\end{tabular}

Table 3: Shear stress values as reported in literature and generated by both 2D and 3D numerical models.

\begin{tabular}{|c|c|c|c|c|c|}
\hline \multicolumn{3}{|c|}{ Testing Conditions } & \multirow[b]{2}{*}{ Data Source } & \multirow[b]{2}{*}{$\begin{array}{l}\text { Wall Shear Stress } \\
{[\mathrm{Pa}]}\end{array}$} & \multirow[b]{2}{*}{$\begin{array}{l}\text { Error } \\
{[\%]}\end{array}$} \\
\hline $\begin{array}{l}\text { Medium Dynamic } \\
\text { Viscosity }[\mathrm{mPa} \cdot \mathrm{s}]\end{array}$ & $\begin{array}{l}\text { Inlet Flow } \\
{[\mu L / h]}\end{array}$ & Rate & & & \\
\hline \multirow{3}{*}{12} & \multirow{3}{*}{\multicolumn{2}{|c|}{1}} & Tanaka et al. (2006) [35] & 0.60 & 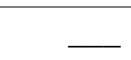 \\
\hline & & & 2D Model & 0.34 & 43.33 \\
\hline & & & 3D Model & 0.59 & 1.67 \\
\hline \multirow{3}{*}{12} & \multirow{3}{*}{\multicolumn{2}{|c|}{10}} & Tanaka et al. (2006) [35] & 6.00 & $\underline{-}$ \\
\hline & & & 2D Model & 3.40 & 43.33 \\
\hline & & & 3D Model & 5.87 & 2.17 \\
\hline \multirow{3}{*}{1} & \multirow{3}{*}{\multicolumn{2}{|c|}{2.5}} & Tanaka et al. (2006) [35] & 0.14 & - \\
\hline & & & 2D Model & 0.07 & 50 \\
\hline & & & 3D Model & 0.12 & 14.29 \\
\hline \multirow{3}{*}{12} & \multirow{3}{*}{\multicolumn{2}{|c|}{2.5}} & Tanaka et al. (2006) [35] & 1.60 & - \\
\hline & & & 2D Model & 0.85 & 47.88 \\
\hline & & & 3D Model & 1.47 & 8.13 \\
\hline
\end{tabular}


Table 4: Overview of Parameters and respective grade of influence on gradient generation. Rank is defined as follows: +++ - Gradient Developer, parameters in which gradient is highly dependant on; ++ - Gradient Refiner, parameters whose influence improves gradient generation; + - Gradient Neutral, parameters that exhibit a very small contribution for gradient modelling. *: Regulating the Heigh of Channels, while presenting an insignificant effect on gradient generation, it is highly important to control shear stress and therefore guarantee correct cell culture and fucntion.

\begin{tabular}{|c|c|c|c|}
\hline Parameter & Rank & Effect & Observation \\
\hline & +++ & Gradient Development & $\begin{array}{l}\text { High rates may cause harmful } \\
\text { conditions for cell culture }\end{array}$ \\
\hline $\begin{array}{l}\text { Width of Serpentine } \\
\text { Channels }\end{array}$ & ++ & $\begin{array}{l}\text { Control mixing of species at } \\
\text { the respective channels }\end{array}$ & $\begin{array}{l}\text { Width should be defined with care to } \\
\text { allow organoid input before testing }\end{array}$ \\
\hline $\begin{array}{l}\text { Number of Serpentine } \\
\text { Loops }\end{array}$ & ++ & $\begin{array}{l}\text { Regulate length for mixing of } \\
\text { species within the respective } \\
\text { channels }\end{array}$ & $\begin{array}{l}\text { An excessive number of loops may } \\
\text { create unnecessary space for mixing }\end{array}$ \\
\hline $\begin{array}{l}\text { Length of Serpentine } \\
\text { Channels }\end{array}$ & ++ & $\begin{array}{l}\text { Regulate length for mixing of } \\
\text { species within the respective } \\
\text { channels }\end{array}$ & $\begin{array}{l}\text { An excessive length may create } \\
\text { unnecessary space for mixing }\end{array}$ \\
\hline $\begin{array}{l}\text { Width of Collecting } \\
\text { Channels }\end{array}$ & ++ & $\begin{array}{l}\text { Control width of gradients } \\
\text { and stability of CLs }\end{array}$ & $\begin{array}{l}\text { Wider channels may cause unstable } \\
\text { gradients }\end{array}$ \\
\hline $\begin{array}{l}\text { Distance Between } \\
\text { Collecting Channel's } \\
\text { Outlets }\end{array}$ & ++ & Define width of gradient & $\begin{array}{l}\text { A wide interval between channels } \\
\text { compromises gradient quality }\end{array}$ \\
\hline Width of Main Chamber & ++ & $\begin{array}{l}\text { Define width and stability of } \\
\text { gradient }\end{array}$ & $\begin{array}{l}\text { A wider chamber may cause unstable } \\
\text { CLs if changed individually }\end{array}$ \\
\hline $\begin{array}{l}\text { Shape of Main Chamber } \\
\text { Outlet }\end{array}$ & ++ & $\begin{array}{l}\text { Control stability of CLs } \\
\text { towards the end of the } \\
\text { chamber }\end{array}$ & $\begin{array}{l}\text { More effective outlet systems usually } \\
\text { add geometric complexity }\end{array}$ \\
\hline Length of Main Chamber & ++ & $\begin{array}{l}\text { Regulate time for particle } \\
\text { convection within the main } \\
\text { chamber }\end{array}$ & $\begin{array}{l}\text { Significantly influences the device's } \\
\text { overall length }\end{array}$ \\
\hline Height of Channels & $+*$ & $\begin{array}{l}\text { Regulate flow induced shear } \\
\text { stress }\end{array}$ & $\begin{array}{l}\text { High shear stress may cause harmful } \\
\text { conditions for cellular growth }\end{array}$ \\
\hline $\begin{array}{l}\text { Length of Inlet/Outlet } \\
\text { Channels }\end{array}$ & + & $\begin{array}{l}\text { Regulate overall length of the } \\
\text { device }\end{array}$ & $\begin{array}{l}\text { An excessive length may create } \\
\text { unnecessary space }\end{array}$ \\
\hline $\begin{array}{l}\text { Distance between Main } \\
\text { Chamber and Serpentine } \\
\text { Channels }\end{array}$ & + & $\begin{array}{l}\text { Regulate overall length of the } \\
\text { device }\end{array}$ & $\begin{array}{l}\text { An excessively low distance may } \\
\text { compromise the gradient }\end{array}$ \\
\hline Distance Between & + & Regulate overall length of the & An excessive distance may over- \\
\hline
\end{tabular}




\begin{tabular}{|c|c|c|}
\hline Serpentine Channels & device & dimension the device \\
\hline Height of Channels [mm] & Qin & Average Surface Shear Stress [Pa] \\
\hline \multirow{4}{*}{0.30} & 1.0 & 0.22 \\
\hline & 1.5 & 0.33 \\
\hline & 2.0 & 0.44 \\
\hline & 2.5 & 0.55 \\
\hline \multirow{4}{*}{0.35} & 1.0 & 0.06 \\
\hline & 1.5 & 0.08 \\
\hline & 2.0 & 0.11 \\
\hline & 2.5 & 0.14 \\
\hline \multirow{4}{*}{0.40} & 1.0 & 0.025 \\
\hline & 1.5 & 0.037 \\
\hline & 2.0 & 0.050 \\
\hline & 2.5 & 0.062 \\
\hline
\end{tabular}

Table 5: Variation of the average cell surface shear stress at the main chamber. 
Table 6: Parameters defining the LGoaC solution.

\begin{tabular}{|c|c|}
\hline Parameter & Value \\
\hline$h \quad h \quad[/ h] \times 103$ & 1.50 \\
\hline$h \quad[/ h] \times 103$ & 1.07 \\
\hline $\mathbf{R}$ & 0.71 \\
\hline $\mathrm{R} h$ & 0.21 \\
\hline Width of CR outer serpentine channels [ ] & 0.30 \\
\hline Number of CR outer serpentine loops & 3 \\
\hline Length of CR outer serpentine channels [ ] & 0.036 \\
\hline Width of CR middle serpentine channels [ ] & 0.565 \\
\hline Number of CR middle serpentine loops & 6 \\
\hline Length of CR middle serpentine channels [ ] & 0.05 \\
\hline Width of serpentine channels [ ] & 0.30 \\
\hline Number of serpentine loops & 3 \\
\hline Length of serpentine channels [ ] & 31.78 \\
\hline Diameter of serpentine loop [ ] & 0.90 \\
\hline Width of collecting channels [ ] & 0.50 \\
\hline Distance between collecting channel's outlets [ ] & 0.15 \\
\hline Width of main chamber [ ] & 3.10 \\
\hline Length of the main chamber [ ] & 8.00 \\
\hline Height of channels [ ] & 0.40 \\
\hline Length of inlet channels [ ] & 3.00 \\
\hline Width of inlet/outlet channels [ ] & 0.30 \\
\hline Length of outlet [ ] & 0.50 \\
\hline Distance between main chamber and serpentines [ ] & 5.00 \\
\hline Distance between serpentine channels [ ] & 4.50 \\
\hline
\end{tabular}

